\title{
SOME REMARKS ON CATEGORIES OF MODULES MODULO MORPHISMS WITH ESSENTIAL KERNEL OR SUPERFLUOUS IMAGE
}

\author{
Adel Alahmadi and Alberto Facchini*
}

\begin{abstract}
For an ideal $\mathcal{I}$ of a preadditive category $\mathcal{A}$, we study when the canonical functor $C: \mathcal{A} \rightarrow \mathcal{A} / \mathcal{I}$ is local. We prove that there exists a largest full subcategory $\mathcal{C}$ of $\mathcal{A}$ for which the canonical functor $C: \mathcal{C} \rightarrow \mathcal{C} / \mathcal{I}$ is local. Under this condition, the functor $C$ turns out to be a weak equivalence between $\mathcal{C}$ and $\mathcal{C} / \mathcal{I}$. If $\mathcal{A}$ is additive (with splitting idempotents), then $\mathcal{C}$ is additive (with splitting idempotents). The category $\mathcal{C}$ is ample in several cases, such as the case when $\mathcal{A}=\operatorname{Mod}-R$ and $\mathcal{I}$ is the ideal $\Delta$ of all morphisms with essential kernel. In this case, the category $\mathcal{C}$ contains, for instance, the full subcategory $\mathcal{F}$ of Mod- $R$ whose objects are all the continuous modules. The advantage in passing from the category $\mathcal{F}$ to the category $\mathcal{F} / \mathcal{I}$ lies in the fact that, although the two categories $\mathcal{F}$ and $\mathcal{F} / \mathcal{I}$ are weakly equivalent, every endomorphism has a kernel and a cokernel in $\mathcal{F} / \Delta$, which is not true in $\mathcal{F}$. In the final section, we extend our theory from the case of one ideal $\mathcal{I}$ to the case of $n$ ideals $\mathcal{I}_{1}, \ldots, \mathcal{I}_{n}$
\end{abstract}

\section{Introduction}

We say that an additive functor $F: \mathcal{A} \rightarrow \mathcal{B}$ between preadditive categories $\mathcal{A}$ and $\mathcal{B}$ is a local functor if, for every morphism $f: A \rightarrow B$ in the category $\mathcal{A}$, $F(f)$ isomorphism in $\mathcal{B}$ implies $f$ isomorphism in $\mathcal{A}[4]$. A functor $F: \mathcal{A} \rightarrow \mathcal{B}$ is isomorphism reflecting if, for every $A, B$ objects of $\mathcal{A}, F(A) \cong F(B)$ implies $A \cong B$. Let $\mathcal{A}$ be a preadditive category and let $\mathcal{I}$ be an ideal of $\mathcal{A}$. The aim of this paper is to study the case when the canonical functor $C: \mathcal{A} \rightarrow \mathcal{A} / \mathcal{I}$ is a local functor. The canonical functor $C: \mathcal{A} \rightarrow \mathcal{A} / \mathcal{I}$ is local if and only if $\mathcal{I}$ is contained in the Jacobson radical $\mathcal{J}$ of $\mathcal{A}$, if and only if (when the endomorphism rings of all non-zero objects of the preadditive category $\mathcal{A}$ are

Received July 2, 2012; Revised August 10, 2012.

2010 Mathematics Subject Classification. 16D90.

Key words and phrases. preadditive category, additive functor, local functor, essential kernel, superfluous image.

* Partially supported by Università di Padova (Progetto di ricerca di Ateneo CPDA105885/10 "Differential graded categories" and Progetto ex 60\% "Anelli e categorie di moduli") 
semilocal rings) every maximal ideal of $\mathcal{A}$ contains $\mathcal{I}$ (Proposition 2.3). The case in which the canonical functor $\mathcal{A} \rightarrow \mathcal{A} / \mathcal{I}_{1} \times \mathcal{A} / \mathcal{I}_{2}$ is local, where $\mathcal{I}_{1}$ and $\mathcal{I}_{2}$ are two ideals of the preadditive category $\mathcal{A}$, was studied in [6].

It turns out that when the functor $C: \mathcal{A} \rightarrow \mathcal{A} / \mathcal{I}$ is local, then $C$ must be necessarily isomorphism reflecting [4, Lemma 3.6(b)]. More precisely, in this case the functor $C$ is a weak equivalence in the sense that it is isomorphism reflecting and dense. For the definitions, see Section 2. Thus when the category $\mathcal{A}$ is additive, the commutative monoids $V(\mathcal{A})$ and $V(\mathcal{A} / \mathcal{I})$, defined on the skeletons of $\mathcal{A}$ and $\mathcal{A} / \mathcal{I}$, respectively, are isomorphic monoids (see Remark 2.2).

In this paper, we prove that given any ideal $\mathcal{I}$ of a preadditive category $\mathcal{B}$, there is a largest full subcategory $\mathcal{C}$ of $\mathcal{B}$ for which the canonical functor $C: \mathcal{C} \rightarrow$ $\mathcal{C} / \mathcal{I}$ is local (Theorem 2.4). Our main application is to the ideal $\Delta$ of Mod- $R$ of all morphisms with essential kernel, where $R$ is any fixed ring (Section 3). In this case, the largest subcategory $\mathcal{E}$ of Mod- $R$ for which the functor $\mathcal{E} \rightarrow$ $\mathcal{E} / \Delta$ is local turns out to be very ample because it contains all semisimple modules, all modules with a local endomorphism ring, all non-singular modules, all indecomposable almost self-injective modules and all continuous modules (In this paper, we use three times the term "ample subcategory". Here, "ample" does not have the meaning it has in algebraic geometry, but the meaning it has in the common language). It is well-known that for every continuous module $A_{R}$, in particular, for every injective $R$-module, one has that $\Delta\left(A_{R}, A_{R}\right)=$ $J\left(\operatorname{End}\left(A_{R}\right)\right)$ and that $\operatorname{End}\left(A_{R}\right) / J\left(\operatorname{End}\left(A_{R}\right)\right)$ is von Neumann regular. Here we study the category $\mathcal{E}$ whose objects are all $R$-modules $A_{R}$ with $\Delta\left(A_{R}, A_{R}\right) \subseteq$ $J\left(\operatorname{End}\left(A_{R}\right)\right)$.

Let $\mathcal{F}$ be the full subcategory of Mod- $R$ whose objects are all continuous right $R$-modules. The case of continuous modules is the most important for us because, via the weak equivalence $C$ between the categories $\mathcal{F}$ and $\mathcal{F} / \Delta$, we give a categorical perspective to the results on continuous modules presented in [11]. We will show that every endomorphism has a kernel and a cokernel in $\mathcal{F} / \Delta$, while this is not true in the category $\mathcal{F}$ (Theorem 3.13 and Example 3.14). These properties hold not only in $\mathcal{F}$ but also in other categories, namely, in any full subcategory of Mod- $R$ on which $\Delta$ is contained in the Jacobson radical and whose objects are modules satisfying Condition $\left(C_{1}\right)$ [11, p. 18].

In Section 4, we dualize our previous results, considering the ideal $\Sigma$ of Mod- $R$ of all morphisms with a superfluous image (Section 4). Note that in [7], it was proved that the canonical functor Mod- $R \rightarrow \operatorname{Mod}-R / \Delta \times \operatorname{Mod}-R / \Sigma$ is always a local functor.

Finally in the last section, we introduce some non-commutative polynomials that allow us to give an explicit description of the inverse of an isomorphism $f$ in $\mathcal{A} / \mathcal{I}_{1} \cap \cdots \cap \mathcal{I}_{n}$ from the inverses of $f$ in the factor categories $\mathcal{A} / \mathcal{I}_{1}, \ldots, \mathcal{A} / \mathcal{I}_{n}$. Here, $\mathcal{I}_{1}, \ldots, \mathcal{I}_{n}$ denote ideals of the preadditive category $\mathcal{A}$ with Jacobson radical $\mathcal{J}$. One obtains that the canonical functor $\mathcal{A} \rightarrow \mathcal{A} / \mathcal{I}_{1} \times \cdots \times \mathcal{A} / \mathcal{I}_{n}$ is local if and only if the functor $\mathcal{A} \rightarrow \mathcal{A} / \mathcal{I}_{1} \cap \cdots \cap \mathcal{I}_{n}$ is local, if and only if $\mathcal{I}_{1} \cap$ $\cdots \cap \mathcal{I}_{n} \subseteq \mathcal{J}$. This has various applications, one of which is the fact that there 
is a largest full subcategory $\mathcal{C}$ for which the functor $\mathcal{C}: \mathcal{C} \rightarrow \mathcal{C} / \mathcal{I}_{1} \times \cdots \times \mathcal{A} / \mathcal{I}_{n}$ is local.

This paper is heavily based on the techniques of [6] and [7]. It is largely an application and a continuation of those articles.

In this paper, the symbol $R$ denotes an associative ring with identity $1 \neq 0$ and $J(R)$ the Jacobson radical of $R$. For any category $\mathcal{A}, \operatorname{Ob}(\mathcal{A})$ denotes the class of all objects of $\mathcal{A}$.

\section{Generalities}

Let $\mathcal{A}$ and $\mathcal{B}$ be preadditive categories and $F: \mathcal{A} \rightarrow \mathcal{B}$ be an additive functor. We say that the functor $F$ is $[4,5]$ :

(1) a local functor if, for every morphism $f: A \rightarrow B$ in $\mathcal{A}, F(f)$ isomorphism in $\mathcal{B}$ implies $f$ isomorphism in $\mathcal{A}$;

(2) an isomorphism reflecting functor if, for every $A, B$ objects of $\mathcal{A}, F(A) \cong$ $F(B)$ implies $A \cong B$;

(3) a dense functor if every object of $\mathcal{B}$ is isomorphic to $F(A)$ for some object $A$ of $\mathcal{A}$;

(4) a weak equivalence if it is isomorphism reflecting and dense.

In particular, every category equivalence $F: \mathcal{A} \rightarrow \mathcal{B}$ is a weak equivalence.

The kernel of any local functor $F: \mathcal{A} \rightarrow \mathcal{B}$ is contained in the Jacobson radical $\mathcal{J}$ of the category $\mathcal{A}[7$, Example 2.1(c)]. Here, the Jacobson radical $\mathcal{J}$ of $\mathcal{A}$ is the ideal defined, for every $A, B \in \mathrm{Ob}(\mathcal{A})$, by $\mathcal{J}(A, B):=\{f \in$ $\operatorname{Hom}_{\mathcal{A}}(A, B) \mid 1_{A}-g f$ has a left inverse (equivalently, a two-sided inverse) for every morphism $g: B \rightarrow A\}$. Conversely, a full functor $F: \mathcal{A} \rightarrow \mathcal{B}$ is a local functor if and only if its kernel is contained in the Jacobson radical $\mathcal{J}$ of $\mathcal{A}[7$, Example 2.1(d)].

Given an ideal $\mathcal{I}$ of a preadditive category $\mathcal{A}$, we can construct the factor category $\mathcal{A} / \mathcal{I}$. The objects of $\mathcal{A} / \mathcal{I}$ are the same objects as $\mathcal{A}$. In the factor category $\mathcal{A} / \mathcal{I}$, the group of all morphisms between two objects $A, B \in \mathrm{Ob}(\mathcal{A})=$ $\operatorname{Ob}(\mathcal{A} / \mathcal{I})$ is $\operatorname{Hom}_{\mathcal{A} / \mathcal{I}}(A, B):=\operatorname{Hom}_{\mathcal{A}}(A, B) / \mathcal{I}(A, B)$. The composition in $\mathcal{A} / \mathcal{I}$ is that induced by the composition in $\mathcal{A}$. There is a canonical functor $C: \mathcal{A} \rightarrow$ $\mathcal{A} / \mathcal{I}$.

It is easily seen [4, Example 3.4 and Lemma 3.6(b)] that local functors need not to be isomorphism reflecting, but that local full functors are isomorphism reflecting. In the next example, we show that there exist canonical functors $C: \mathcal{A} \rightarrow \mathcal{A} / \mathcal{I}$ that are isomorphism reflecting full functors, but not local functors.

Example 2.1. Let $\mathcal{A}$ be the full subcategory of Ab whose objects are all finitely generated free abelian groups and let $p$ be a prime number. Let $\mathcal{I}$ be the ideal of $\mathcal{A}$ defined, for every $G, H \in \mathrm{Ob}(\mathcal{A})$, by $\mathcal{I}(G, H):=p \operatorname{Hom}(G, H)$. From $J(\mathbb{Z})=$ 0 , it follows easily that the Jacobson radical $\mathcal{J}$ of $\mathcal{A}$ is the zero radical. Thus $\mathcal{I} \nsubseteq \mathcal{J}$ so that the canonical full functor $C: \mathcal{A} \rightarrow \mathcal{A} / \mathcal{I}$ is not local. In order to 
show that this functor $C$ is isomorphism reflecting, let $G \cong \mathbb{Z}^{n}$ and $H \cong \mathbb{Z}^{m}$ be objects of $\mathcal{A}$ isomorphic in $\mathcal{A} / \mathcal{I}$. Then there exist morphisms $f: G \rightarrow H$ and $g: H \rightarrow G$ with $g f-1_{G} \in p \operatorname{Hom}(G, H)$, and similarly for $f g$. Applying the functor $-\otimes_{\mathbb{Z}} \mathbb{Z} / p \mathbb{Z}$, we find that $(g \otimes \mathbb{Z} / p \mathbb{Z})(f \otimes \mathbb{Z} / p \mathbb{Z})-1_{G \otimes \mathbb{Z} / p \mathbb{Z}}=0$, and similarly for $f g$. Thus $G \otimes \mathbb{Z} / p \mathbb{Z} \cong \mathbb{Z} / p \mathbb{Z}^{n}$ is isomorphic to $H \otimes \mathbb{Z} / p \mathbb{Z} \cong \mathbb{Z} / p \mathbb{Z}^{m}$. It follows that $n=m$ and $G \cong H$.

We want to determine, for a full subcategory $\mathcal{A}$ of a preadditive category $\mathcal{B}$ and an ideal $\mathcal{I}$ of $\mathcal{B}$, when the canonical functor $C: \mathcal{A} \rightarrow \mathcal{A} / \mathcal{I}$ is local, that is, when a morphism $f$ in $\mathcal{A}$ with $C(f)=\bar{f}$ isomorphism in $\mathcal{A} / \mathcal{I}$ is necessarily an isomorphism in $\mathcal{A}$. As we have already remarked, every local full functor is isomorphism reflecting so that when the canonical functor $C: \mathcal{A} \rightarrow \mathcal{A} / \mathcal{I}$ is local, it is a weak equivalence. This means that the image via $C$ of a skeleton $V(\mathcal{A})$ of $\mathcal{A}$ is a skeleton $V(\mathcal{A} / \mathcal{I})$ of $\mathcal{A} / \mathcal{I}$, and that $C$ induces a bijection between the two skeletons $V(\mathcal{A})$ and $V(\mathcal{A} / \mathcal{I})$. These facts are our main motivation for the study of when the canonical functor $C: \mathcal{A} \rightarrow \mathcal{A} / \mathcal{I}$ is local because if $C$ is local, then the two categories are weakly equivalent, hence with isomorphic skeletons, but working in $\mathcal{A} / \mathcal{I}$ can sometimes be easier than working in the category $\mathcal{A}$. In the next remark, we describe the situation in a more precise way.

Remark 2.2. The setting can be presented in the language of commutative monoids. Let $\mathcal{I}$ be an ideal of an additive category $\mathcal{A}$. Assume that the canonical functor $C: \mathcal{A} \rightarrow \mathcal{A} / \mathcal{I}$ is local and that idempotents split in both categories $\mathcal{A}$ and $\mathcal{A} / \mathcal{I}$. If $A$ is an object of $\mathcal{A}$, then we can construct the full subcategory add $\operatorname{Ad}_{\mathcal{A}}(A)$ of $\mathcal{A}$ whose objects are all direct summands of $A^{n}$ for some $n \geq 0$. Similarly, we can construct the full subcategory $\operatorname{add}_{\mathcal{A} / \mathcal{I}}(A)$ of $\mathcal{A} / \mathcal{I}$. For instance, if $R$ is a ring and $\mathcal{A}$ is the category Mod- $R$, the subcategory $\operatorname{add}_{\operatorname{Mod}-R}\left(R_{R}\right)$ is the category, usually denoted by proj- $R$, whose objects are all finitely generated projective right $R$-modules. The full categories $\operatorname{add}_{\mathcal{A}}(A)$ and $\operatorname{add}_{\mathcal{A} / \mathcal{I}}(A)$ are additive categories in which idempotents split. For any additive category $\mathcal{C}$, it is possible to define a commutative monoid structure on any skeleton $V(\mathcal{C})$ of $\mathcal{C}$. If $A \mapsto\langle A\rangle$ is the mapping $\mathrm{Ob}(\mathcal{C}) \rightarrow V(\mathcal{C})$ that associates to any object $A$ of $\mathcal{C}$ the unique object $\langle A\rangle$ of $V(\mathcal{C})$ isomorphic to $A$, then the operation on $V(\mathcal{C})$ is defined by $\langle A\rangle+\langle B\rangle=\langle A \oplus B\rangle$ for any pair $A, B$ of objects. Thus $V(\mathcal{C})$ becomes an additive commutative monoid, possibly large when $\mathcal{C}$ is not skeletally small. The monoid $V(\operatorname{proj}-R)$ for a $\operatorname{ring} R$ is usually indicated as $V(R)$. Every additive functor $F: \mathcal{C} \rightarrow \mathcal{C}^{\prime}$ between additive categories $\mathcal{C}$ and $\mathcal{C}^{\prime}$ induces a monoid homomorphism $V(F): V(\mathcal{C}) \rightarrow V\left(\mathcal{C}^{\prime}\right)$, which assigns to each element $\langle A\rangle$ of $V(\mathcal{C})$ the element $\langle F(A)\rangle$ of $V\left(\mathcal{C}^{\prime}\right)$.

In our case, the fact that $C: \mathcal{A} \rightarrow \mathcal{A} / \mathcal{I}$ is local, hence a weak equivalence, has as a consequence that the monoid homomorphism $V(C): V(\mathcal{A}) \rightarrow V(\mathcal{A} / \mathcal{I})$ is a monoid isomorphism. Thus if $A$ is an object of $\mathcal{C}$, then the monoids $V\left(\operatorname{add}_{\mathcal{A}}(A)\right)$ and $V\left(\operatorname{add}_{\mathcal{A} / \mathcal{I}}(A)\right)$ are isomorphic monoids (More precisely, the mapping $V\left(\operatorname{add}_{\mathcal{A}}(A)\right) \rightarrow V\left(\operatorname{add}_{\mathcal{A} / \mathcal{I}}(A)\right)$ is onto because if $B$ is isomorphic to a 
direct summand of $A^{n}$ in $\mathcal{A} / \mathcal{I}$, then there exists $D \in \mathrm{Ob}(\mathcal{A} / \mathcal{I})=\mathrm{Ob}(\mathcal{A})$ such that $B \oplus D \cong A$ in $\mathcal{A} / \mathcal{I}$ due to the fact that idempotents split in $\mathcal{A} / \mathcal{I}$. But $\mathcal{A}$ is additive and $C$ is an isomorphism reflecting functor so that $B \oplus D$ exists in $\mathcal{A}$ and is isomorphic to $A^{n}$ in $\mathcal{A}$. This proves that the mapping $V\left(\operatorname{add}_{\mathcal{A}}(A)\right) \rightarrow$ $V\left(\operatorname{add}_{\mathcal{A} / \mathcal{I}}(A)\right)$ is onto). If $R$ denotes the endomorphism $\operatorname{ring} \operatorname{End}_{\mathcal{A}}(A)$ of $A$ in $\mathcal{A}$ and $I:=\mathcal{I}(A, A)$ so that $R / I$ is the endomorphism ring of $A$ in $\mathcal{A} / \mathcal{I}$, then the functor $\operatorname{Hom}_{\mathcal{C} / \mathcal{I}}(A,-): \mathcal{C} / \mathcal{I} \rightarrow \operatorname{Mod}-R / I$ induces an equivalence $\operatorname{add}_{\mathcal{A} / \mathcal{I}}(A) \rightarrow$ $\operatorname{proj}-R / I\left[6\right.$, Lemma 3.1]. Thus the three monoids $V\left(\operatorname{add}_{\mathcal{A}}(A)\right), V\left(\operatorname{add}_{\mathcal{A} / \mathcal{I}}(A)\right)$ and $V(R / I)$ are isomorphic when the functor $C: \mathcal{A} \rightarrow \mathcal{A} / \mathcal{I}$ is local.

Recall that a maximal ideal [6] of a preadditive category $\mathcal{A}$ is an ideal of $\mathcal{A}$ that is properly contained only in the improper ideal $\operatorname{Hom}_{\mathcal{A}}$ of $\mathcal{A}$. A ring $S$ is semilocal if $S / J(S)$ is semisimple Artinian. A preadditive category $\mathcal{A}$ is null if all its objects are zero objects. We say that a preadditive category is semilocal if it is non-null and the endomorphism ring of every non-zero object is a semilocal ring [6, Definition 4.2]. We trivially get the following result from [7, Proposition 3.1] for the case in which the two ideals in the statement of the proposition coincide.

Proposition 2.3. Let $\mathcal{I}$ be an ideal of a preadditive category $\mathcal{A}$.

(a) If the canonical functor $\mathcal{A} \rightarrow \mathcal{A} / \mathcal{I}$ is a local functor, then every maximal ideal of $\mathcal{A}$ contains $\mathcal{I}$.

(b) If the category $\mathcal{A}$ is semilocal and every maximal ideal of $\mathcal{A}$ contains $\mathcal{I}$, then the canonical functor $\mathcal{A} \rightarrow \mathcal{A} / \mathcal{I}$ is local.

Notice that Proposition 2.3(b) does not hold without the hypothesis of $\mathcal{A}$ being semilocal. For instance, let $\mathcal{A}$ be the category Mod- $k$, where $k$ is any division ring, and let $\mathcal{I}$ be the ideal of Mod- $k$ consisting of all linear transformations of finite rank. Then Mod- $k$ does not have maximal ideals and the canonical functor $\mathcal{A} \rightarrow \mathcal{A} / \mathcal{I}$ is not local.

As we have already mentioned, we want to determine, for a full subcategory $\mathcal{A}$ of a preadditive category $\mathcal{B}$ and an ideal $\mathcal{I}$ of $\mathcal{B}$, when the canonical functor $C: \mathcal{A} \rightarrow \mathcal{A} / \mathcal{I}$ is local. As $C$ is a full functor, this is equivalent to requiring that the kernel $\mathcal{I}$ of $C$ be contained in the Jacobson radical $\mathcal{J}$ of $\mathcal{A}$. In particular, we must have $\mathcal{I}(A, A) \subseteq J\left(\operatorname{End}_{\mathcal{B}}(A)\right)$ for every object $A$ of $\mathcal{A}$. In the next theorem, we show that the full subcategory $\mathcal{C}$ of $\mathcal{B}$ whose objects are all the objects $A$ of $\mathcal{B}$ with $\mathcal{I}(A, A) \subseteq J\left(\operatorname{End}_{\mathcal{B}}(A)\right)$ is the largest full subcategory of $\mathcal{B}$ for which the functor $C: \mathcal{C} \rightarrow \mathcal{C} / \mathcal{I}$ is local.

Theorem 2.4. Let $\mathcal{I}$ be an ideal of a preadditive category $\mathcal{B}$. Let $\mathcal{C}$ be the full subcategory of $\mathcal{B}$ whose objects are the objects $A$ of $\mathcal{B}$ with $\mathcal{I}(A, A) \subseteq$ $J\left(\operatorname{End}_{\mathcal{B}}(A)\right)$. Then, on the category $\mathcal{C}$, the ideal $\mathcal{I}$ is contained in the Jacobson radical $\mathcal{J}$, so that the canonical functor $C: \mathcal{C} \rightarrow \mathcal{C} / \mathcal{I}$ is local. Moreover, the category $\mathcal{C}$ is the largest full subcategory of $\mathcal{B}$ with this property. Finally, if $\mathcal{B}$ is an additive category, then $\mathcal{C}$ is an additive category, and if $\mathcal{B}$ is additive and idempotents split in $\mathcal{B}$, then idempotents split also in $\mathcal{C}$. 
Proof. In order to show that, in the category $\mathcal{C}$, the ideal $\mathcal{I}$ is contained in the Jacobson radical $\mathcal{J}$, we must prove that $\mathcal{I}(A, B) \subseteq \mathcal{J}(A, B)$ for every pair of objects $A, B$ of $\mathcal{C}$. Now, if $f \in \mathcal{I}(A, B)$ and $g: B \rightarrow A$ is any morphism, then $g f \in \mathcal{I}(A, A) \subseteq J(\operatorname{End}(A))$ so that $1_{A}-g f$ is an automorphism of $A$. Thus $f \in \mathcal{J}(A, B)$ and $\mathcal{I} \subseteq \mathcal{J}$ on $\mathcal{C}$. It is now clear that $\mathcal{C}$ is the largest full subcategory of Mod- $R$ with this property.

Assume $\mathcal{B}$ is additive. To prove that the full subcategory $\mathcal{C}$ of $\operatorname{Mod}-R$ is additive, we must show the class of all objects $A$ of $\mathcal{B}$ with $\mathcal{I}(A, A) \subseteq J\left(\operatorname{End}_{\mathcal{B}}(A)\right)$ is closed under finite coproducts. Now

$$
\operatorname{End}_{\mathcal{B}}(A \oplus B)=\left(\begin{array}{cc}
\operatorname{End}_{\mathcal{B}}(A) & \operatorname{Hom}_{\mathcal{B}}(B, A) \\
\operatorname{Hom}_{\mathcal{B}}(A, B) & \operatorname{End}_{\mathcal{B}}(B)
\end{array}\right)
$$

and

$$
\mathcal{K}(A \oplus B, A \oplus B)=\left(\begin{array}{ll}
\mathcal{K}(A, A) & \mathcal{K}(B, A) \\
\mathcal{K}(A, B) & \mathcal{K}(B, B)
\end{array}\right)
$$

for every ideal $\mathcal{K}$ of $\mathcal{C}$. Thus if $A$ and $B$ are such that $\mathcal{I}(A, A) \subseteq J\left(\operatorname{End}_{\mathcal{B}}(A)\right)$ and $\mathcal{I}(B, B) \subseteq J\left(\operatorname{End}_{\mathcal{B}}(B)\right)$, then we can conclude that $\mathcal{I}(A \oplus B, A \oplus B) \subseteq$ $J\left(\operatorname{End}_{\mathcal{B}}(A \oplus B)\right)$ by what we have seen in the previous paragraph.

Finally, assume that $\mathcal{B}$ is additive and idempotents split in $\mathcal{B}$. Let $f: C \rightarrow C$ be an idempotent endomorphism in $\mathcal{C}$. Then $C \in \mathrm{Ob}(\mathcal{C})$ and there exist an object $B \in \mathrm{Ob}(\mathcal{B})$ and morphisms $g: C \rightarrow B$ and $h: B \rightarrow C$ such that $f=h g$. Then $C=B \oplus K$ for a suitable object $K$ of $\mathcal{B}$ [4, Lemma 2.1]. By similar arguments as in the previous paragraph, we get that $\mathcal{I}(B \oplus K, B \oplus K) \subseteq$ $J\left(\operatorname{End}_{\mathcal{B}}(B \oplus K)\right)$ implies $\mathcal{I}(B, B) \subseteq J\left(\operatorname{End}_{\mathcal{B}}(B)\right)$.

Remark 2.5. Let $A$ be an object of $\mathcal{B}$ and assume that the endomorphism ring $\operatorname{End}_{\mathcal{B}}(A)$ of $A$ is local so that, in particular, $A \neq 0$. Let $\mathcal{I}$ be any ideal of $\mathcal{B}$ and $\mathcal{C}$ be the category considered in Theorem 2.4. Then two cases can occur: either $\mathcal{I}(A, A)$ is a proper ideal of $\operatorname{End}_{\mathcal{B}}(A)$ or it is the improper ideal. If $\mathcal{I}(A, A)$ is a proper ideal of $\operatorname{End}_{\mathcal{B}}(A)$, then $\mathcal{I}(A, A) \subseteq J\left(\operatorname{End}_{\mathcal{B}}(A)\right)$ so that $A$ is an object of $\mathcal{C}$. If $\mathcal{I}(A, A)=\operatorname{End}_{\mathcal{B}}(A)$, that is, $\mathcal{I}(A, A)$ is the improper ideal, then $\mathcal{I}(A, A) \nsubseteq J\left(\operatorname{End}_{\mathcal{B}}(A)\right)$ so that $A$ is not an object of $\mathcal{C}$. Thus an object $A$ of $\mathcal{B}$ is an object of $\mathcal{C}$ if and only if the ideal $\mathcal{I}(A, A)$ is a proper ideal of $\operatorname{End}_{\mathcal{B}}(A)$.

\section{The ideal of morphisms with essential kernel}

Let $R$ be a ring and Mod- $R$ be the category of all right $R$-modules. Setting $\Delta\left(A_{R}, B_{R}\right):=\left\{f: A_{R} \rightarrow B_{R} \mid\right.$ ker $f$ essential in $\left.A_{R}\right\}$ for every pair of right modules $A_{R}, B_{R}$, we get an ideal $\Delta$ of Mod- $R$ and, correspondingly, the factor category Mod- $R / \Delta$ and the canonical functor $C: \operatorname{Mod}-R \rightarrow \operatorname{Mod}-R / \Delta$.

In [7, Proposition 4.5], it is shown that if two modules $A_{R}, B_{R}$ are isomorphic objects in the category Mod- $R / \Delta$, then they have the same monogeny class, that is, there are a monomorphism of $A_{R}$ into $B_{R}$ and a monomorphism of $B_{R}$ into $A_{R}$. Recall that a module is uniform if it has Goldie dimension one. 
Lemma 3.1. If $A_{R}$ is a uniform right $R$-module, then $\Delta\left(A_{R}, A_{R}\right)$ is a completely prime two-sided ideal of $\operatorname{End}\left(A_{R}\right)$ so that the endomorphism ring of $A_{R}$ in the category Mod- $R / \Delta$ is a (non-necessarily commutative) integral domain.

Proof. If the module $A_{R}$ is uniform, then the ideal $\Delta\left(A_{R}, A_{R}\right)$ consists of all endomorphisms of $A_{R}$ that are not monomorphisms. Thus $\Delta\left(A_{R}, A_{R}\right)$ is a completely prime ideal by $[3$, Lemma $6.26(\mathrm{a})]$. In particular, $\operatorname{End}_{\operatorname{Mod}-R / \Delta}\left(A_{R}\right)=$ $\operatorname{End}\left(A_{R}\right) / \Delta\left(A_{R}, A_{R}\right)$ is a domain.

Lemma 3.1 does not hold when the module $A_{R}$ is not uniform. To see this, it suffices to take a division ring $R$, a two-dimensional vector space $A_{R}$ and two non-zero endomorphisms of $A_{R}$ whose composition is zero. In this case, $\Delta\left(A_{R}, B_{R}\right)$ is the zero ideal of $\operatorname{End}\left(A_{R}\right)$, which is not completely prime, and $\operatorname{End}\left(A_{R}\right)$ is not a domain.

As we have already stated, we want to determine when, for a full subcategory $\mathcal{A}$ of Mod- $R$, the canonical functor $C: \mathcal{A} \rightarrow \mathcal{A} / \Delta$ is local. As $C$ is a full functor, this is equivalent to requiring that the kernel $\Delta$ of $C$ be contained in the Jacobson radical $\mathcal{J}$ of $\mathcal{A}$. In particular, we must have $\Delta\left(A_{R}, A_{R}\right) \subseteq$ $J\left(\operatorname{End}\left(A_{R}\right)\right)$ for every object $A_{R}$ of $\mathcal{A}$. In the next proposition, we characterize the $R$-modules $A_{R}$ with this property.

Proposition 3.2. Let $A_{R}$ be a right module over a ring $R$ and let $E\left(A_{R}\right)$ be the injective envelope of $A_{R}$. The following conditions are equivalent:

(a) $\Delta\left(A_{R}, A_{R}\right) \subseteq J\left(\operatorname{End}\left(A_{R}\right)\right)$.

(b) If $g$ is an endomorphism of $A_{R}$ and there exists an essential submodule $B_{R}$ of $A_{R}$ for which $g(b)=b$ for all $b \in B_{R}$, then $g$ is an automorphism of $A_{R}$.

(c) If $h$ is an automorphism of $E\left(A_{R}\right)$ with $h\left(A_{R}\right) \subseteq A_{R}$ and there exists an essential submodule $B_{R}$ of $A_{R}$ for which $h(b)=b$ for all $b \in B_{R}$, then $h\left(A_{R}\right)=A_{R}$.

Proof. (a) $\Longrightarrow$ (b) Assume that (a) holds. Let $g$ be an endomorphism of $A_{R}$ and let $B_{R}$ be an essential submodule of $A_{R}$ with $g(b)=b$ for all $b \in B_{R}$. Then $g-1$ has essential kernel, and hence belongs to $\Delta\left(A_{R}, A_{R}\right)$ so that $g$ is an automorphism of $A_{R}$ by (a).

(b) $\Longrightarrow$ (c) Suppose that (b) holds. Let $h$ be an automorphism of $E\left(A_{R}\right)$ with $h\left(A_{R}\right) \subseteq A_{R}$. Assume that there exists an essential submodule $B_{R}$ of $A_{R}$ for which $h(b)=b$ for all $b \in B_{R}$. Then it is possible to apply (b) to the restriction $g$ of $h$ to $A_{R}$. By (b), $g$ is an automorphism of $A_{R}$ so that $h\left(A_{R}\right)=g\left(A_{R}\right)=A_{R}$.

(c) $\Longrightarrow$ (a) Let $f$ be a morphism in $\Delta\left(A_{R}, A_{R}\right)$ and $f^{\prime}$ be any other endomorphism of $A_{R}$. Let $h \in \operatorname{End}\left(E\left(A_{R}\right)\right)$ be an extension to $E\left(A_{R}\right)$ of $1-f^{\prime} f: A_{R} \rightarrow$ $A_{R}$. Then $h$ is an endomorphism of $E\left(A_{R}\right)$ with $h\left(A_{R}\right)=\left(1-f^{\prime} f\right)\left(A_{R}\right) \subseteq A_{R}$ and $h$ is the identity on the essential submodule ker $f$ of $A_{R}$. In particular, 
$h: E\left(A_{R}\right) \rightarrow E\left(A_{R}\right)$ is a monomorphism. The image of $h$ is therefore an injective submodule of $E\left(A_{R}\right)$ isomorphic to $E\left(A_{R}\right)$ and contains $B_{R}$. Thus $h\left(E\left(A_{R}\right)\right)=E\left(A_{R}\right)$. We can now apply (c) to the automorphism $h$ of $E\left(A_{R}\right)$, which yields that $h\left(A_{R}\right)=A_{R}$. So the restriction $1-f^{\prime} f$ of $h$ to $A_{R}$ is an automorphism of $A_{R}$. It follows that $f$ is in the Jacobson radical of the ring $\operatorname{End}\left(A_{R}\right)$.

From Theorem 2.4, we have:

Theorem 3.3. Let $\mathcal{E}$ be the full subcategory of Mod- $R$ whose objects are all right $R$-modules satisfying the equivalent conditions of Proposition 3.2. Then in the category $\mathcal{E}$, the ideal $\Delta$ is contained in the Jacobson radical $\mathcal{J}$ so that the canonical functor $C: \mathcal{E} \rightarrow \mathcal{E} / \Delta$ is local. The category $\mathcal{E}$ is the largest full subcategory of Mod- $R$ with this property. Moreover, $\mathcal{E}$ is an additive category in which idempotents split.

Now we will show that the category $\mathcal{E}$ described in Theorem 3.3 is rather ample. Let us begin by proving that all modules with a local endomorphism ring satisfy the equivalent conditions of Proposition 3.2.

Proposition 3.4. The following conditions are equivalent for a right $R$-module $A_{R}$ :

(a) $A_{R}$ has a local endomorphism ring $\operatorname{End}\left(A_{R}\right)$ in $\operatorname{Mod}-R$.

(b) $A_{R}$ satisfies the equivalent conditions of Proposition 3.2 and has a local endomorphism ring in $\operatorname{Mod}-R / \Delta$.

Proof. (a) $\Longrightarrow$ (b) Let $A_{R}$ be a module with $\operatorname{End}\left(A_{R}\right)$ local so that, in particular, $A_{R} \neq 0$. Thus $1_{R} \notin \Delta\left(A_{R}, A_{R}\right)$. It follows that the proper ideal $\Delta\left(A_{R}, A_{R}\right)$ of $\operatorname{End}\left(A_{R}\right)$ is contained in the maximal ideal $J\left(\operatorname{End}\left(A_{R}\right)\right)$ of $\operatorname{End}\left(A_{R}\right)$. Hence $A_{R}$ satisfies the conditions of Proposition 3.2. The rest of the proof of the implication (a) $\Longrightarrow(\mathrm{b})$ is trivial because if $A_{R}$ has a local endomorphism ring $\operatorname{End}\left(A_{R}\right)$ in $\operatorname{Mod}-R$, then $\operatorname{End}_{\operatorname{Mod}-R / \Delta}\left(A_{R}\right)=\operatorname{End}\left(A_{R}\right) / \Delta\left(A_{R}, A_{R}\right)$ is also local.

(b) $\Longrightarrow$ (a) Assume $\operatorname{End}\left(A_{R}\right) / \Delta\left(A_{R}, A_{R}\right)$ is local and that $A_{R}$ satisfies the equivalent conditions of Proposition 3.2. Then $\Delta\left(A_{R}, A_{R}\right) \subseteq J\left(\operatorname{End}\left(A_{R}\right)\right)$ so that $J\left(\operatorname{End}\left(A_{R}\right) / \Delta\left(A_{R}, A_{R}\right)\right)=J\left(\operatorname{End}\left(A_{R}\right)\right) / \Delta\left(A_{R}, A_{R}\right)$. It follows that

$\operatorname{End}\left(A_{R}\right) / J\left(\operatorname{End}\left(A_{R}\right)\right) \cong\left(\operatorname{End}\left(A_{R}\right) / \Delta\left(A_{R}, A_{R}\right)\right) / J\left(\operatorname{End}\left(A_{R}\right) / \Delta\left(A_{R}, A_{R}\right)\right)$.

Hence $\operatorname{End}\left(A_{R}\right) / J\left(\operatorname{End}\left(A_{R}\right)\right)$ is a division ring and $\operatorname{End}\left(A_{R}\right)$ is local.

Proposition 3.5. Every non-singular $R$-module satisfies the equivalent conditions of Proposition 3.2.

Proof. Let $A_{R}$ be a non-singular module. Let $g$ be an endomorphism of $A_{R}$ such that there exists an essential submodule $B_{R}$ of $A_{R}$ with $g(b)=b$ for all $b \in B_{R}$. Then $\operatorname{ker}(1-g)$ is an essential submodule of $A_{R}$, and $1-g$ induces 
a morphism $\overline{1-g}: A_{R} / \operatorname{ker}(1-g) \rightarrow A_{R}$. Now $A_{R} / \operatorname{ker}(1-g)$ is a singular module because $\operatorname{ker}(1-g)$ is essential in $A_{R}$, and $A_{R}$ is non-singular. Thus

$$
\operatorname{Hom}\left(A_{R} / \operatorname{ker}(1-g), A_{R}\right)=0
$$

so that $\overline{1-g}=\overline{0}$. It follows that $(1-g)\left(A_{R}\right)=0$, and therefore, $g=1$ is an automorphism.

Proposition 3.6. Let $A_{R}$ be an $R$-module and let $\operatorname{soc}\left(A_{R}\right)$ be its socle. If $\operatorname{Hom}\left(A_{R} / \operatorname{soc}\left(A_{R}\right), A_{R}\right)=0$, then $A_{R}$ satisfies the equivalent conditions of Proposition 3.2 .

Proof. Assume that $\operatorname{Hom}\left(A_{R} / \operatorname{soc}\left(A_{R}\right), A_{R}\right)=0$. We will prove that $\Delta\left(A_{R}\right.$, $\left.A_{R}\right)=0$. If $f \in \Delta\left(A_{R}, A_{R}\right)$, then ker $f$ is an essential submodule. As the socle is the intersection of all essential submodules, we have that $\operatorname{ker} f \supseteq \operatorname{soc}\left(A_{R}\right)$. Thus $f$ induces a morphism $\bar{f}: A_{R} / \operatorname{soc}\left(A_{R}\right) \rightarrow A_{R}$. From $\operatorname{Hom}\left(A_{R} / \operatorname{soc}\left(A_{R}\right)\right.$, $\left.A_{R}\right)=0$, it follows that $\bar{f}$, hence $f$, is zero. This proves that $\Delta\left(A_{R}, A_{R}\right)=0$ so that $\Delta\left(A_{R}, A_{R}\right) \subseteq J\left(\operatorname{End}\left(A_{R}\right)\right)$.

As an immediate corollary, we get that:

Corollary 3.7. Every semisimple R-module satisfies the equivalent conditions of Proposition 3.2.

We say that an $R$-module is uniform if it has Goldie dimension one, couniform (or hollow) if it has dual Goldie dimension one, and biuniform if it is uniform and couniform [3]. In particular, every biuniform module is non-zero and indecomposable. For instance, non-zero uniserial modules are biuniform and if $R$ is a local ring, then every cyclic submodule of an indecomposable injective $R$-module is biuniform.

We begin with couniform modules.

Proposition 3.8. Let $A_{R}$ be a couniform module, $\operatorname{End}\left(A_{R}\right)$ its endomorphism ring, $\Delta\left(A_{R}, A_{R}\right)$ the ideal of $\operatorname{End}\left(A_{R}\right)$ consisting of all endomorphisms with an essential kernel and $\Sigma\left(A_{R}, A_{R}\right)$ the ideal of $\operatorname{End}\left(A_{R}\right)$ consisting of all endomorphisms with a superfluous image. The following conditions are equivalent:

(a) $A_{R}$ satisfies the equivalent conditions of Proposition 3.2 .

(b) $\Delta\left(A_{R}, A_{R}\right)+\Sigma\left(A_{R}, A_{R}\right)$ is a proper ideal of $\operatorname{End}\left(A_{R}\right)$.

Proof. (a) $\Longrightarrow$ (b) If $\Delta\left(A_{R}, A_{R}\right)+\Sigma\left(A_{R}, A_{R}\right)$ is not a proper ideal, then there exist $f \in \Delta\left(A_{R}, A_{R}\right)$ and $g \in \Sigma\left(A_{R}, A_{R}\right)$ with $f+g=1_{A}$. The morphism $g$ does not satisfy Condition (b) of Proposition 3.2 because ker $f$ is an essential submodule of $A_{R}$ for which $g(x)=x$ for all $x \in \operatorname{ker} f$, but $g$ is not an automorphism of $A_{R}$.

(b) $\Longrightarrow$ (a) Assume that Condition (b) of Proposition 3.2 does not hold. Then there exist an endomorphism $g$ of $A_{R}$ and an essential submodule $B_{R}$ of $A_{R}$ for which $g(b)=b$ for all $b \in B_{R}$, but $g$ is not an automorphism of $A_{R}$. Then $\operatorname{ker}(1-g)$ is an essential submodule of $A_{R}$ and $g$ is a monomorphism, but 
not an epimorphism. As $A_{R}$ is couniform, it follows that $g \in \Sigma\left(A_{R}, A_{R}\right)$ and $1-g \in \Delta\left(A_{R}, A_{R}\right)$. Thus $\Delta\left(A_{R}, A_{R}\right)+\Sigma\left(A_{R}, A_{R}\right)$ is the improper ideal.

Proposition 3.9. Let $A_{R}$ be a couniform module, let $\operatorname{Max}\left(A_{R}\right)$ be the set of all maximal (two-sided) ideals of the endomorphism ring $\operatorname{End}\left(A_{R}\right), \mathcal{D}:=\{M \in$ $\left.\operatorname{Max}\left(A_{R}\right) \mid \Delta\left(A_{R}, A_{R}\right) \subseteq M\right\}$ and $\mathcal{S}:=\left\{M \in \operatorname{Max}\left(A_{R}\right) \mid \Sigma\left(A_{R}, A_{R}\right) \subseteq M\right\}$. Then

(a) $\operatorname{Max}\left(A_{R}\right)=\mathcal{D} \cup \mathcal{S}$.

(b) $\mathcal{D} \cap \mathcal{S} \neq \emptyset$ if and only if $A_{R}$ satisfies the equivalent conditions of Proposition 3.2 .

The same properties hold if one substitutes $\operatorname{Max}\left(A_{R}\right)$ with $\mathrm{r}-\operatorname{Max}\left(A_{R}\right)$, the set of all maximal right ideals of $\operatorname{End}\left(A_{R}\right)$, or $\mathrm{l}-\operatorname{Max}\left(A_{R}\right)$, the set of all maximal left ideals of $\operatorname{End}\left(A_{R}\right)$.

Proof. Every simple ring is primitive so that every maximal ideal is both left and right primitive. Thus every maximal ideal contains the Jacobson radical. By [7, Corollary 4.4], $\Delta\left(A_{R}, A_{R}\right) \cap \Sigma\left(A_{R}, A_{R}\right) \subseteq J\left(\operatorname{End}\left(A_{R}\right)\right)$. Every simple ring is a prime ring so that every maximal ideal is a prime ideal. Thus if $M \in \operatorname{Max}\left(A_{R}\right)$, then $\Delta\left(A_{R}, A_{R}\right) \Sigma\left(A_{R}, A_{R}\right) \subseteq \Delta\left(A_{R}, A_{R}\right) \cap \Sigma\left(A_{R}, A_{R}\right) \subseteq$ $J\left(\operatorname{End}\left(A_{R}\right)\right) \subseteq M$ implies that either $\Delta\left(A_{R}, A_{R}\right) \subseteq M$ or $\Sigma\left(A_{R}, A_{R}\right) \subseteq M$. This proves (a).

For (b), we have that $\mathcal{D} \cap \mathcal{S}=\emptyset$ if and only if there is no maximal ideal of $\operatorname{End}\left(A_{R}\right)$ containing both $\Delta\left(A_{R}, A_{R}\right)$ and $\Sigma\left(A_{R}, A_{R}\right)$, that is, if and only if $\Delta\left(A_{R}, A_{R}\right)+\Sigma\left(A_{R}, A_{R}\right)$ is the improper ideal of $\operatorname{End}\left(A_{R}\right)$. Proposition 3.8 allows us to conclude for $\operatorname{Max}\left(A_{R}\right)$.

As far as r-Max $\left(A_{R}\right)$ is concerned, let $M_{R}$ be a maximal right ideal of $\operatorname{End}\left(A_{R}\right)$. For (a), we must prove that either $\Delta\left(A_{R}, A_{R}\right) \subseteq M_{R}$ or $\Sigma\left(A_{R}, A_{R}\right) \subseteq$ $M_{R}$. Now $R_{R} / M_{R}$ is a simple right $R$-module so that its annihilator $P$ is a right primitive ideal contained in $M_{R}$. Thus $P$ is a prime ideal [10, Proposition 3.15] and $P \supseteq J\left(\operatorname{End}\left(A_{R}\right)\right) \supseteq \Delta\left(A_{R}, A_{R}\right) \cap \Sigma\left(A_{R}, A_{R}\right) \supseteq \Delta\left(A_{R}, A_{R}\right) \Sigma\left(A_{R}, A_{R}\right)$, so we can conclude the proof of (a) as before. The proof of (b) is similar to that for maximal two-sided ideals in the previous paragraph.

Let $A_{R}$ be a biuniform right $R$-module and let $E:=\operatorname{End}\left(A_{R}\right)$ be its endomorphism ring. Let $I$ be the subset of $E$ whose elements are all the endomorphisms of $A_{R}$ that are not monomorphisms, and $K$ be the subset of $E$ whose elements are all the endomorphisms of $A_{R}$ that are not epimorphisms. Then $I$ and $K$ are two two-sided completely prime ideals of $E$, and every proper right ideal of $E$ and every proper left ideal of $E$ is contained either in $I$ or in $K$ [3, Theorem 9.1]. Notice that $I=\Delta\left(A_{R}, A_{R}\right)$ and $K=\Sigma\left(A_{R}, A_{R}\right)$. For any biuniform module $A_{R}$, exactly one of the following two conditions hold: either $I$ and $K$ are comparable, that is, $I \subseteq K$ or $K \subseteq I$, and in this case, $E$ is a local ring and $I \cup K=I+K$ is its maximal ideal; or $I$ and $K$ are not comparable, $J(E)=I \cap K$, and $E / J(E)$ is canonically isomorphic to the direct product of the two division rings $E / I$ and $E / K$. 
Proposition 3.10. A biuniform $R$-module $A_{R}$ satisfies the equivalent conditions of Proposition 3.2 if and only if its endomorphism ring $\operatorname{End}\left(A_{R}\right)$ is a local ring.

Proof. Let $A_{R}$ be a biuniform module. Then $\operatorname{End}\left(A_{R}\right)$ is local if and only if $I$ and $K$ are comparable ideals, that is, if and only if $I+K$ is a proper ideal of $\operatorname{End}\left(A_{R}\right)$. By Proposition 3.8, this occurs if and only if $A_{R}$ satisfies the equivalent conditions of Proposition 3.2.

As far as the almost self-injective modules studied in [1] are concerned, we have the following proposition. Recall that a ring $S$ is said to be a left chain ring if the left $S$-module ${ }_{S} S$ is uniserial.

Proposition 3.11. Let $A_{R}$ be an indecomposable almost self-injective $R$-module. Then $A_{R}$ satisfies the equivalent conditions of Proposition 3.2, $\Delta\left(A_{R}, A_{R}\right)$ is a completely prime ideal of $\operatorname{End}\left(A_{R}\right)$, and $\operatorname{End}\left(A_{R}\right) / \Delta\left(A_{R}, A_{R}\right)$ is a left chain domain.

Proof. The module $A_{R}$ is uniform by $[1$, Lemma 1$]$ so that $\Delta\left(A_{R}, A_{R}\right)$ is completely prime and $\operatorname{End}\left(A_{R}\right) / \Delta\left(A_{R}, A_{R}\right)$ is a domain by Lemma 3.1. Moreover, $S:=\operatorname{End}\left(A_{R}\right)$ is a local ring [1, Theorem 5] so that, by Proposition 3.4, $A_{R}$ satisfies the equivalent conditions of Proposition 3.2.

To conclude the proof, it remains to show that if $g, h \in S$, then either $S g+\Delta\left(A_{R}, A_{R}\right) \subseteq S h+\Delta\left(A_{R}, A_{R}\right)$ or $S h+\Delta\left(A_{R}, A_{R}\right) \subseteq S g+\Delta\left(A_{R}, A_{R}\right)$. Now if $g$ and $h$ are monomorphisms, then $\operatorname{ker} g=\operatorname{ker} h$ so that either $S g \subseteq$ $S h$ or $S h \subseteq S g$ [1, Lemma 6(ii)], and we are done. If one of $g$ and $h$ is a monomorphism and the other is not, then we conclude by [1, Lemma 6(ii)]. If both $g$ and $h$ are not monomorphisms, then $g, h \in \Delta\left(A_{R}, A_{R}\right)$ so that $S g+$ $\Delta\left(A_{R}, A_{R}\right)=S h+\Delta\left(A_{R}, A_{R}\right)=\Delta\left(A_{R}, A_{R}\right)$.

Clearly, any cohopfian module, that is, any module for which every injective endomorphism is an automorphism, satisfies Condition (b) of Proposition 3.2. In particular, every Artinian $R$-module belongs to the category $\mathcal{E}$ of the statement of Theorem 3.3.

As far as the regular module $R_{R}$ is concerned, notice that $\Delta\left(R_{R}, R_{R}\right)$ corresponds to the right singular ideal $Z\left(R_{R}\right)$ of the ring $R$. Thus the module $R_{R}$ satisfies the equivalent conditions of Proposition 3.2 if and only if $Z\left(R_{R}\right) \subseteq J(R)$. In particular, if either $R$ is local or $R$ is any right nonsingular ring, then $R$ belongs to the category $\mathcal{E}$ (Theorem 3.3).

Recall that a module $A_{R}$ is continuous if: $\left(C_{1}\right)$ every submodule of $A_{R}$ is essential in a direct summand of $A_{R}$; and $\left(C_{2}\right)$ if a submodule $B$ of $A_{R}$ is isomorphic to a direct summand of $A_{R}$, then $B$ is a direct summand of $A_{R}$. Every direct summand of a continuous module is a continuous module, but $A_{R}$ continuous does not imply $A_{R} \oplus A_{R}$ continuous in general [11, Proposition 2.7 and Corollary 2.11]. It is well-known that for any continuous module $A_{R}$, one 
has $\Delta\left(A_{R}, A_{R}\right)=J\left(\operatorname{End}\left(A_{R}\right)\right), \operatorname{End}\left(A_{R}\right) / \Delta\left(A_{R}, A_{R}\right)$ is a von Neumann regular ring and idempotents modulo $\Delta\left(A_{R}, A_{R}\right)$ can be lifted [11, Proposition 3.5 and Lemma 3.7]. Thus:

Proposition 3.12. Every continuous $R$-module $A_{R}$ satisfies the equivalent conditions of Proposition 3.2, and the endomorphism ring of the object $A_{R}$ in the factor category Mod- $R / \Delta$ is von Neumann regular.

As a corollary, we have that every injective $R$-module satisfies the equivalent conditions of Proposition 3.2. A quasi-continuous module is a module $A_{R}$ satisfying Condition $\left(C_{1}\right)$ above and Condition $\left(C_{3}\right)$ : If $B_{1}$ and $B_{2}$ are direct summands of $A_{R}$ such that $B_{1} \cap B_{2}=0$, then $B_{1} \oplus B_{2}$ is a direct summand of $A_{R}$. There exist quasi-continuous modules that do not satisfy the equivalent conditions of Proposition 3.2. For instance, we have seen in Proposition 3.10 that every biuniform module whose endomorphism ring is not local does not satisfy the equivalent conditions of Proposition 3.2, but a biuniform module is uniform, hence quasi-continuous [11, Proposition 2.5].

For modules that satisfy Condition $\left(C_{1}\right)$, we have:

Theorem 3.13. Let $R$ be any ring and $\mathcal{A}$ be a full subcategory of Mod- $R$. Assume that idempotent splits in $\mathcal{A}$ and that all the objects of $\mathcal{A}$ are modules $A_{R}$ that satisfy Condition $\left(C_{1}\right)$. Then every morphism has kernel and cokernel in $\mathcal{A} / \Delta$. In particular, idempotents split in $\mathcal{A} / \Delta$.

Proof. Let $f: A_{R} \rightarrow B_{R}$ be a morphism in $\mathcal{A}$ and $\bar{f}: A_{R} \rightarrow B_{R}$ be its image in $\mathcal{A} / \Delta$. We must prove that $\bar{f}$ has a kernel and a cokernel in $\mathcal{A} / \Delta$. Now the kernel ker $f$ of $f$ in Mod- $R$ is essential in a direct summand $A_{1}$ of $A_{R}$ by Condition $\left(C_{1}\right)$. Let $A_{2}$ be a complement of $A_{1}$ so that $A_{R}=A_{1} \oplus A_{2}$. Thus $f: A_{R}=A_{1} \oplus A_{2} \rightarrow B_{R}$ can be written in the form $f=\left(f_{1}, f_{2}\right)$ with each $f_{i}: A_{i} \rightarrow B_{R}$ being a module morphism. Clearly, $\bar{f}=\overline{\left(0, f_{2}\right)}$ because the difference $f-\left(0, f_{2}\right)=\left(f_{1}, 0\right)$ has essential kernel ker $f \oplus A_{2}$ in $A_{R}$. We leave to the reader to show that if $\varepsilon_{1}: A_{1} \rightarrow A_{R}$ is the inclusion, then $\overline{\varepsilon_{1}}$ is the kernel of $\bar{f}=\overline{\left(0, f_{2}\right)}$ in the category $\mathcal{A} / \Delta$.

Now $f_{2}\left(A_{2}\right)$ is essential in a direct summand $B_{1}$ of $B_{R}$ so that $B_{R}=B_{1} \oplus B_{2}$ for some submodule $B_{2}$ of $B_{R}$. Let $q: B_{R}=B_{1} \oplus B_{2} \rightarrow B_{2}$ be the canonical projection of $B_{R}$ onto $B_{2}$ with kernel $B_{1}$. We will now show that $\bar{q}: B_{R} \rightarrow B_{2}$ is the cokernel of $\bar{f}=\overline{\left(0, f_{2}\right)}$ in $\mathcal{A} / \Delta$. Clearly, $q\left(0, f_{2}\right)=0$ so that $\overline{q f}=\overline{0}$. Let $g: B_{R} \rightarrow C_{R}$ be any other morphism in $\mathcal{A}$ with $\overline{g f}=\overline{0}$. Then $g$ can be written in the form $g=\left(g_{1}, g_{2}\right)$ with each $g_{i}: B_{i} \rightarrow C_{R}$ being a morphism in $\mathcal{A}$, the morphism $\bar{q}$ becomes in matrix form $\overline{\left(0,1_{B_{2}}\right)}$, and $\left(0, f_{2}\right): A_{R}=$ $A_{1} \oplus A_{2} \rightarrow B_{R}=B_{1} \oplus B_{2}$ can be written in matrix form as $\left(0, f_{2}\right)=\left(\begin{array}{cc}0 & f_{2}^{\prime} \\ 0 & 0\end{array}\right)$ with $f_{2}^{\prime}: A_{2} \rightarrow B_{1}$ being an $R$-module monomorphism since $f_{2}: A_{2} \rightarrow B_{R}$ is an injective mapping (Notice that $A_{2}$ has zero intersection with the kernel ker $f$ of $f$ in Mod- $R$ ). Then $\overline{0}=\overline{g f}=\overline{\left(g_{1}, g_{2}\right)\left(\begin{array}{cc}0 & f_{2}^{\prime} \\ 0 & 0\end{array}\right)}=\overline{\left(0, g_{1} f_{2}^{\prime}\right)}$. Thus $g_{1} f_{2}^{\prime}: A_{2} \rightarrow C_{R}$ has essential kernel. Equivalently, $\operatorname{ker} g_{1} \cap f_{2}^{\prime}\left(A_{2}\right)$ is an essential submodule 
of $f_{2}^{\prime}\left(A_{2}\right)$. But $f_{2}^{\prime}\left(A_{2}\right)=f_{2}\left(A_{2}\right)$ is essential in $B_{1}$ so that ker $g_{1}$ is essential in $B_{1}$, that is, $\overline{g_{1}}=\overline{0}$. Thus $\bar{g}=\overline{\left(g_{1}, g_{2}\right)}=\overline{\left(0, g_{2}\right)}$ factors uniquely through $\bar{q}=\overline{\left(0,1_{B_{2}}\right)}$.

Finally, if $\bar{e}: A_{R} \rightarrow A_{R}$ is an idempotent in $\mathcal{A} / \Delta$ and $\bar{k}$, with $k: K_{R} \rightarrow A_{R}$, is a kernel of the idempotent $\overline{1_{A_{R}}-e}$ in $\mathcal{A} / \Delta$, then $\overline{\left(1_{A_{R}}-e\right)} \bar{e}=0$ implies that there exists a unique morphism $\bar{g}: A_{R} \rightarrow K_{R}$ such that $\bar{e}=\bar{k} \bar{g}$. As $\overline{\left(1_{A_{R}}-e\right) k}=\overline{0}$, it follows that $\bar{k}=\overline{e k}=\overline{k g k}$. But kernels are monomorphisms, whence $\overline{1_{K_{R}}}=\overline{g k}$.

Theorem 3.13 applies to the full subcategory $\mathcal{F}$ of Mod- $R$ whose objects are all continuous right $R$-modules. The rest of this section is devoted to some remarks about this category $\mathcal{F}$. First of all, notice that the weak equivalence $C: \mathcal{F} \rightarrow \mathcal{F} / \Delta$ does not preserve kernels nor cokernels, in general. For instance, the Prüfer group $\mathbb{Z}\left(p^{\infty}\right)$ is a continuous $\mathbb{Z}$-module, and the kernel both in Mod- $R$ and in $\mathcal{F}$ of the morphism $\lambda_{p}: \mathbb{Z}\left(p^{\infty}\right) \rightarrow \mathbb{Z}\left(p^{\infty}\right)$ given by left multiplication by $p$ is the embedding $\varepsilon: \mathbb{Z} / p \mathbb{Z} \hookrightarrow \mathbb{Z}\left(p^{\infty}\right)$. Modulo $\Delta$, we find that $\overline{\lambda_{p}}$ is the zero endomorphism of $\mathbb{Z}\left(p^{\infty}\right)$ so that its kernel in $\mathcal{F} / \Delta$ is the identity mapping $\mathbb{Z}\left(p^{\infty}\right) \rightarrow \mathbb{Z}\left(p^{\infty}\right)$. But $\mathbb{Z}\left(p^{\infty}\right)$ and $\mathbb{Z} / p \mathbb{Z}$ are not isomorphic in $\mathcal{F} / \Delta$. Hence $C$ does not preserve kernels. As far as cokernels are concerned, the group $\mathbb{Z} / p^{2} \mathbb{Z}$ is a continuous $\mathbb{Z}$-module, and the cokernel both in Mod- $R$ and in $\mathcal{F}$ of the morphism $\lambda_{p}: \mathbb{Z} / p^{2} \mathbb{Z} \rightarrow \mathbb{Z} / p^{2} \mathbb{Z}$ given by left multiplication by $p$ is the canonical projection $\pi: \mathbb{Z} / p^{2} \mathbb{Z} \rightarrow \mathbb{Z} / p \mathbb{Z}$. Modulo $\Delta$, the morphism $\overline{\lambda_{p}}$ is the zero endomorphism of $\mathbb{Z} / p^{2} \mathbb{Z}$ so that its cokernel in $\mathcal{F} / \Delta$ is the identity mapping $\mathbb{Z} / p^{2} \mathbb{Z} \rightarrow \mathbb{Z} / p^{2} \mathbb{Z}$. But $\mathbb{Z} / p \mathbb{Z}$ and $\mathbb{Z} / p^{2} \mathbb{Z}$ are not isomorphic in $\mathcal{F} / \Delta$. Hence $C$ does not preserve cokernels.

Example 3.14. By Theorem 3.13, kernels and cokernels always exist in the category $\mathcal{F} / \Delta$. On the contrary, there are morphisms in the category $\mathcal{F}$ that do not have kernels nor cokernels in $\mathcal{F}$. For instance, consider the endomorphism $f$ of $\mathbb{Z}\left(p^{\infty}\right)^{2}$ given by the left multiplication by $\left(\begin{array}{ll}p & 0 \\ 0 & p^{2}\end{array}\right)$. We will prove that $f$ does not have a kernel in $\mathcal{F}$.

Assume the contrary. Let $k: K \rightarrow \mathbb{Z}\left(p^{\infty}\right)^{2}$ be a kernel of $f$ in $\mathcal{F}$, where $K$ is a suitable continuous $\mathbb{Z}$-module. Then $f k=0$ so that the image $k(K)$ of $k$ is contained in the subgroup $\mathbb{Z} / p \mathbb{Z} \oplus \mathbb{Z} / p^{2} \mathbb{Z}$ of $\mathbb{Z}\left(p^{\infty}\right)^{2}$. Therefore, there is a factorization $k=\varepsilon k^{\prime}$ in the category $\mathrm{Ab}$ of abelian groups, where $k^{\prime}: K \rightarrow$ $\mathbb{Z} / p \mathbb{Z} \oplus \mathbb{Z} / p^{2} \mathbb{Z}$ is a suitable group morphism and $\varepsilon: \mathbb{Z} / p \mathbb{Z} \oplus \mathbb{Z} / p^{2} \mathbb{Z} \rightarrow \mathbb{Z}\left(p^{\infty}\right)^{2}$ is the embedding.

Let $\varepsilon_{1}: \mathbb{Z} / p \mathbb{Z} \rightarrow \mathbb{Z} / p \mathbb{Z} \oplus \mathbb{Z} / p^{2} \mathbb{Z}$ be the inclusion into the first component so that $\varepsilon \varepsilon_{1}: \mathbb{Z} / p \mathbb{Z} \rightarrow \mathbb{Z}\left(p^{\infty}\right)^{2}$ is a morphism in $\mathcal{F}$ with $f \varepsilon \varepsilon_{1}=0$. As $k$ is a kernel of $f$, there exists a unique morphism $g_{1}: \mathbb{Z} / p \mathbb{Z} \rightarrow K$ with $k g_{1}=\varepsilon \varepsilon_{1}$. Then $\varepsilon k^{\prime} g_{1}=\varepsilon \varepsilon_{1}$, and so $k^{\prime} g_{1}=\varepsilon_{1}$. Compose this equality with the first canonical projection $\pi_{1}: \mathbb{Z} / p \mathbb{Z} \oplus \mathbb{Z} / p^{2} \mathbb{Z} \rightarrow \mathbb{Z} / p \mathbb{Z}$, yielding $\pi_{1} k^{\prime} g_{1}=\pi_{1} \varepsilon_{1}=1_{\mathbb{Z} / p \mathbb{Z}}$. Thus $g_{1}: \mathbb{Z} / p \mathbb{Z} \rightarrow K$ is a splitting monomorphism so that $K$ has a direct summand $K_{1}$ isomorphic to $\mathbb{Z} / p \mathbb{Z}$. 
Similarly for the second component. Let $\varepsilon_{2}: \mathbb{Z} / p^{2} \mathbb{Z} \rightarrow \mathbb{Z} / p \mathbb{Z} \oplus \mathbb{Z} / p^{2} \mathbb{Z}$ be the inclusion into the second component, whence $f \varepsilon \varepsilon_{2}=0$. There exists a unique morphism $g_{2}: \mathbb{Z} / p^{2} \mathbb{Z} \rightarrow K$ with $k g_{2}=\varepsilon \varepsilon_{2}$. Then $k^{\prime} g_{2}=\varepsilon_{2}$ so that $\pi_{2} k^{\prime} g_{2}=\pi_{2} \varepsilon_{2}=1_{\mathbb{Z} / p^{2} \mathbb{Z}}$. Thus $K$ has a direct summand $K_{2}$ isomorphic to $\mathbb{Z} / p^{2} \mathbb{Z}$.

The intersection $K_{1} \cap K_{2}$ is a subgroup of the simple group $K_{1}$, and therefore either $K_{1} \subseteq K_{2}$ or $K_{1} \cap K_{2}=0$. Now $K_{1}$ and $K_{2}$ are direct summands of $K$ so that $K_{1} \subseteq K_{2}$ implies that $K_{1}$ is a direct summand of $K_{2}$, which is a contradiction because $\mathbb{Z} / p^{2} \mathbb{Z}$ does not have direct summands isomorphic to $\mathbb{Z} / p \mathbb{Z}$. Thus $K_{1} \cap K_{2}=0$. By [11, Proposition 2.2], $K_{1} \oplus K_{2}$ is a direct summand of $K$ (Condition $\left(C_{3}\right)$ ). But $K$ is continuous so that $K_{1} \oplus K_{2} \cong \mathbb{Z} / p \mathbb{Z} \oplus \mathbb{Z} / p^{2} \mathbb{Z}$ is a continuous abelian group, which is a contradiction. This proves that $f$ does not have a kernel in $\mathcal{F}$.

Similarly, it is possible to prove that the embedding $e_{1}: \mathbb{Z} / p \mathbb{Z} \rightarrow\left(\mathbb{Z} / p^{2} \mathbb{Z}\right)^{2}$ into the first component is a morphism in $\mathcal{F}$ that does not have a cokernel in $\mathcal{F}$.

\section{The ideal of morphisms with superfluous image}

Now we will dualize most of the previous results. Let $R$ be a ring. Set

$$
\Sigma\left(A_{R}, B_{R}\right):=\left\{f: A_{R} \rightarrow B_{R} \mid f\left(A_{R}\right) \text { is superfluous in } B_{R}\right\}
$$

for every pair $A_{R}, B_{R}$ of right modules. Then $\Sigma$ is an ideal of Mod- $R$. There is a canonical functor $C$ of Mod- $R$ onto the factor category $\operatorname{Mod}-R / \Sigma$.

If two modules $A_{R}, B_{R}$ are isomorphic objects in Mod- $R / \Sigma$, then they have the same epigeny class, that is, there are an epimorphism of $A_{R}$ onto $B_{R}$ and an epimorphism of $B_{R}$ onto $A_{R}$ [7, Proposition 4.6].

Lemma 4.1. If $A_{R}$ is a couniform right $R$-module, then $\Sigma\left(A_{R}, A_{R}\right)$ is a completely prime two-sided ideal of $\operatorname{End}\left(A_{R}\right)$ so that the endomorphism ring of $A_{R}$ in the category Mod- $R / \Sigma$ is an integral domain.

Proof. If the module $A_{R}$ is couniform, then the ideal $\Sigma\left(A_{R}, A_{R}\right)$ consists of all endomorphisms of $A_{R}$ that are not epimorphisms. Thus $\Sigma\left(A_{R}, A_{R}\right)$ is completely prime by $[3$, Lemma $6.26(\mathrm{~b})]$. Thus

$$
\operatorname{End}_{\operatorname{Mod}-R / \Sigma}\left(A_{R}\right)=\operatorname{End}\left(A_{R}\right) / \Sigma\left(A_{R}, A_{R}\right)
$$

is a domain.

We want to determine when, for a full subcategory $\mathcal{A}$ of Mod- $R$, the canonical functor $C: \mathcal{A} \rightarrow \mathcal{A} / \Sigma$ is local. As $C$ is a full functor, this is equivalent to requiring that the kernel $\Sigma$ of $C$ be contained in the Jacobson radical $\mathcal{J}$ of $\mathcal{A}$. In the next proposition, we characterize the $R$-modules $A_{R}$ with $\Sigma\left(A_{R}, A_{R}\right) \subseteq J\left(\operatorname{End}\left(A_{R}\right)\right)$.

Proposition 4.2. The following conditions are equivalent for a right module $A_{R}$ over a ring $R$ : 
(a) $\Sigma\left(A_{R}, A_{R}\right) \subseteq J\left(\operatorname{End}\left(A_{R}\right)\right)$.

(b) If $g$ is an endomorphism of $A_{R}$ with a superfluous image and $g(a)=a$ for some $a \in A_{R}$, then $a=0$.

Proof. (a) $\Longrightarrow$ (b) Assume (a) holds. Let $g$ be an endomorphism of $A_{R}$ with a superfluous image and $a$ an element of $A_{R}$ with $g(a)=a$. Then $g \in \Sigma\left(A_{R}, A_{R}\right)$, so $g \in J\left(\operatorname{End}\left(A_{R}\right)\right)$ by (a), and hence $1-g$ is an automorphism of $A_{R}$. Thus $a$, which is an element in the kernel of $1-g$, must be zero.

(b) $\Longrightarrow$ (a) Let $f$ be a morphism in $\Sigma\left(A_{R}, A_{R}\right)$ and $f^{\prime}$ any other endomorphism of $A_{R}$. Assume that (b) holds. We must prove that $1-f^{\prime} f$ is an automorphism of $A_{R}$. Now $f^{\prime} f \in \Sigma\left(A_{R}, A_{R}\right)$ so that $f^{\prime} f\left(A_{R}\right)$ is superfluous in $A_{R}$. Thus $A_{R} \subseteq f^{\prime} f\left(A_{R}\right)+\left(1-f^{\prime} f\right)\left(A_{R}\right)$ implies $A_{R}=\left(1-f^{\prime} f\right)\left(A_{R}\right)$, that is, $1-f^{\prime} f$ is an epimorphism. By (b), $f^{\prime} f(a)=a$ implies $a=0$, that is, $\left(1-f^{\prime} f\right)(a)=0$ implies $a=0$. Thus $1-f^{\prime} f$ is also a monomorphism so that it is an automorphism of $A_{R}$. It follows that $f$ belongs to the Jacobson radical of the ring $\operatorname{End}\left(A_{R}\right)$.

The next result follows immediately from Theorem 2.4.

Theorem 4.3. Let $\mathcal{D}$ be the full subcategory of $\operatorname{Mod}-R$ whose objects are all right $R$-modules satisfying the equivalent conditions of Proposition 4.2. Then in the category $\mathcal{D}$, the ideal $\Sigma$ is contained in the Jacobson radical $\mathcal{J}$ so that the canonical functor $C: \mathcal{D} \rightarrow \mathcal{D} / \Sigma$ is local. The category $\mathcal{D}$ is the largest full subcategory of Mod- $R$ with this property. Moreover, $\mathcal{D}$ is an additive category in which idempotents split.

We want to show also that in this case, the category $\mathcal{D}$ as described in Theorem 4.3 is broad.

Proposition 4.4. The following conditions are equivalent for a right $R$-module $A_{R}$ :

(a) $A_{R}$ has a local endomorphism ring $\operatorname{End}\left(A_{R}\right)$ in $\operatorname{Mod}-R$.

(b) $A_{R}$ satisfies the equivalent conditions of Proposition 4.2 and has a local endomorphism ring in Mod- $R / \Sigma$.

Proof. (a) $\Longrightarrow$ (b) Let $A_{R}$ be a module with $\operatorname{End}\left(A_{R}\right)$ local so that, in particular, $A_{R} \neq 0$. Then either $\Sigma\left(A_{R}, A_{R}\right) \subseteq J\left(\operatorname{End}\left(A_{R}\right)\right)$ for which case $A_{R}$ satisfies the equivalent conditions of Proposition 4.2, or $\Sigma\left(A_{R}, A_{R}\right)$ is the improper ideal of $\operatorname{End}\left(A_{R}\right)$. In the latter case, $1_{A} \in \Sigma\left(A_{R}, A_{R}\right)$ so that $A_{R}$ is superfluous in $A_{R}$, that is, $A_{R}=0$, a contradiction. The rest is trivial because $\operatorname{End}\left(A_{R}\right)$ local implies that its homomorphic image $\operatorname{End}_{\operatorname{Mod}-R / \Sigma}\left(A_{R}\right)=\operatorname{End}\left(A_{R}\right) / \Sigma\left(A_{R}, A_{R}\right)$ is also local.

The proof of $(b) \Longrightarrow(a)$ is similar to the proof of $(b) \Longrightarrow$ (a) in Proposition 3.4.

Recall that a module $A_{R}$ is quasi-projective if, for every module $B_{R}$, every epimorphism $h: A_{R} \rightarrow B_{R}$ and every homomorphism $\ell: A_{R} \rightarrow B_{R}$, there exists an endomorphism $g: A_{R} \rightarrow A_{R}$ with $\ell=h g$. 
Proposition 4.5. Every quasi-projective $R$-module satisfies the equivalent conditions of Proposition 4.2.

Proof. Let $A_{R}$ be a quasi-projective $R$-module. We want to show that

$$
\Sigma\left(A_{R}, A_{R}\right) \subseteq J\left(\operatorname{End}\left(A_{R}\right)\right) .
$$

Since $J\left(\operatorname{End}\left(A_{R}\right)\right)$ is the largest superfluous right ideal of $\operatorname{End}\left(A_{R}\right)$, it suffices to show that if $f \in \Sigma\left(A_{R}, A_{R}\right)$, then $f \operatorname{End}\left(A_{R}\right)$ is a superfluous right ideal of $\operatorname{End}\left(A_{R}\right)$. Hence let $I$ be a right ideal of $\operatorname{End}\left(A_{R}\right)$ and suppose $f \operatorname{End}\left(A_{R}\right)+I=$ $\operatorname{End}\left(A_{R}\right)$. Then there exist $g \in \operatorname{End}\left(A_{R}\right)$ and $h \in I$ with $f g+h=1_{A}$. It follows that $a=f g(a)+h(a)$ for every $a \in A$ so that $A_{R} \subseteq f g\left(A_{R}\right)+h\left(A_{R}\right)$. Now $f g \in$ $\Sigma\left(A_{R}, A_{R}\right)$, and thus $f g\left(A_{R}\right)$ is superfluous in $A_{R}$. Therefore, $A_{R}=h\left(A_{R}\right)$, i.e., $h: A_{R} \rightarrow A_{R}$ is an epimorphism. As $A_{R}$ is quasi-projective, there exists an endomorphism $g: A_{R} \rightarrow A_{R}$ with $1_{A}=h g$. Then $1_{A} \in h \operatorname{End}\left(A_{R}\right) \subseteq I$. It follows that $I=\operatorname{End}\left(A_{R}\right)$, and we can conclude that $f \operatorname{End}\left(A_{R}\right)$ is a superfluous right ideal.

In particular, projective $R$-modules satisfy the equivalent conditions of Proposition 4.2. For the ring $R$, one has $\Sigma\left(R_{R}, R_{R}\right)=J(R)$.

Let us return to arbitrary modules. Recall that the radical $\operatorname{rad}\left(A_{R}\right)$ of a module $A_{R}$ is the intersection of all maximal submodules of $A_{R}$ and that it coincides with the sum of all superfluous submodules of $A_{R}$.

Proposition 4.6. Every $R$-module $A_{R}$ with $\operatorname{Hom}\left(A_{R}, \operatorname{rad}\left(A_{R}\right)\right)=0$ satisfies the equivalent conditions of Proposition 4.2.

Proof. Let $A_{R}$ be an $R$-module with $\operatorname{Hom}\left(A_{R}, \operatorname{rad}\left(A_{R}\right)\right)=0, g$ be an endomorphism of $A_{R}$ with a superfluous image, and $a$ an element of $A_{R}$ with $g(a)=a$. Since $\operatorname{rad}\left(A_{R}\right)$ is equal to the sum of all superfluous submodules of $A_{R}$, we have that $g\left(A_{R}\right) \subseteq \operatorname{rad}\left(A_{R}\right)$. Thus $\operatorname{Hom}\left(A_{R}, \operatorname{rad}\left(A_{R}\right)\right)=0$ implies $\operatorname{Hom}\left(A_{R}, g\left(A_{R}\right)\right)=0$ so that $g=0$. Thus $0=g(a)=a$, as desired.

As the radical of a semisimple module is zero, we immediately get as a corollary that:

Proposition 4.7. Every semisimple $R$-module satisfies the equivalent conditions of Proposition 4.2.

The proofs of Propositions 3.8, 3.9 and 3.10 can be easily dualized, which yields:

Proposition 4.8. Let $A_{R}$ be a uniform module and $\operatorname{End}\left(A_{R}\right)$ be its endomorphism ring. The following conditions are equivalent:

(a) $A_{R}$ satisfies the equivalent conditions of Proposition 4.2.

(b) $\Delta\left(A_{R}, A_{R}\right)+\Sigma\left(A_{R}, A_{R}\right)$ is a proper ideal of $\operatorname{End}\left(A_{R}\right)$.

Proposition 4.9. Let $A_{R}$ be a uniform module, let $\operatorname{Max}\left(A_{R}\right)$ be the set of all maximal (two-sided) ideals of the endomorphism ring $\operatorname{End}\left(A_{R}\right), \mathcal{D}:=\{M \in$ 
$\left.\operatorname{Max}\left(A_{R}\right) \mid \Delta\left(A_{R}, A_{R}\right) \subseteq M\right\}$ and $\mathcal{S}:=\left\{M \in \operatorname{Max}\left(A_{R}\right) \mid \Sigma\left(A_{R}, A_{R}\right) \subseteq M\right\}$. Then

(a) $\operatorname{Max}\left(A_{R}\right)=\mathcal{D} \cup \mathcal{S}$.

(b) $\mathcal{D} \cap \mathcal{S} \neq \emptyset$ if and only if $A_{R}$ satisfies the equivalent conditions of Proposition 4.2 .

Proposition 4.10. A biuniform $R$-module $A_{R}$ satisfies the equivalent conditions of Proposition 4.2 if and only if its endomorphism ring $\operatorname{End}\left(A_{R}\right)$ is a local ring.

Every hopfian module, that is, every module for which all surjective endomorphisms are automorphisms, satisfies Condition (b) of Proposition 4.2. For instance, every Noetherian $R$-module is in the category $\mathcal{D}$ of the statement of Theorem 4.3.

Recall that a module $A_{R}$ is discrete if: $\left(D_{1}\right)$ for every submodule $B$ of $A_{R}$, there is a decomposition $A_{R}=A_{1} \oplus A_{2}$ in which $A_{1}$ is contained in $B$ and $B \cap A_{2}$ is a superfluous submodule of $A_{R}$; and $\left(D_{2}\right)$ if $B$ is a submodule of $A_{R}$ and $A_{R} / B$ is isomorphic to a direct summand of $A_{R}$, then $B$ is a direct summand of $A_{R}$. Every direct summand of a discrete module is a discrete module [11, Lemma 4.7]. It is well-known that for every discrete module $A_{R}$, one has $\Sigma\left(A_{R}, A_{R}\right)=J\left(\operatorname{End}\left(A_{R}\right)\right), \operatorname{End}\left(A_{R}\right) / \Sigma\left(A_{R}, A_{R}\right)$ is a von Neumann regular ring and idempotents modulo $\Sigma\left(A_{R}, A_{R}\right)$ can be lifted [11, Lemma 5.3 and Theorem 5.4]. Thus:

Proposition 4.11. Every discrete R-module $A_{R}$ satisfies the equivalent conditions of Proposition 4.2, and the endomorphism ring of the object $A_{R}$ in the factor category Mod- $R / \Sigma$ is von Neumann regular.

As a corollary, we have that every direct sum of couniform projective modules satisfies the equivalent conditions of Proposition 4.2 [11, Corollary 4.54].

Remark 4.12. There exists a number of classes of modules whose endomorphism rings have only one or two maximal right ideals [8]. This is the case, for instance, of the class of biuniform modules, which we have already considered in this paper, and the class of cyclically presented modules over local rings, that is, the modules isomorphic to $R / a R$ for some $a \in R$. If $A_{R}$ is a module and $\operatorname{End}\left(A_{R}\right)$ has at most two maximal right ideals $M_{1}$ and $M_{2}$ (possibly, $\left.M_{1}=M_{2}\right)$, then for both $i=1,2$, either $M_{i} \supseteq \Delta\left(A_{R}, A_{R}\right)$ or $M_{i} \supseteq \Sigma\left(A_{R}, A_{R}\right)$ [7, Proposition 3.1 and Theorem 4.3].

For instance, it is well-known that if $A_{R}$ is a biuniform module, then $A_{R}$ has at most two maximal ideals, namely, $I:=\left\{f \in \operatorname{End}\left(A_{R}\right) \mid f\right.$ is not a monomorphism $\}$ and $K:=\left\{f \in \operatorname{End}\left(A_{R}\right) \mid f\right.$ is not an epimorphism $\}[3$, Theorem 9.1]. In this case, one precisely has that $I=\Delta\left(A_{R}, A_{R}\right)$ and $K=$ $\Sigma\left(A_{R}, A_{R}\right)$.

But this does not hold for all modules whose endomorphism ring has at most two maximal right ideals. Let $a$ be a non-zero non-invertible element of 
a local $\operatorname{ring} R$ and set $A_{R}:=R / a R$. Let $E:=\{r \in R \mid r a \in a R\}$ be the idealizer of $a R$ so that $\operatorname{End}_{R}(R / a R) \cong E / a R$. Then $\operatorname{End}\left(A_{R}\right)$ has at most two maximal right ideals, which can be either $I / a R$ or $K / a R$, where $I:=\{r \in$ $R \mid r a \in a J(R)\}$ and $K:=J(R) \cap E[2]$. Thus by [7, Proposition 3.1 and Theorem 4.3], we must have one of the two possible cases $I / a R \supseteq \Delta\left(A_{R}, A_{R}\right)$ or $I / a R \supseteq \Sigma\left(A_{R}, A_{R}\right)$, and one of the two possible cases $K / a R \supseteq \Delta\left(A_{R}, A_{R}\right)$ or $K / a R \supseteq \Sigma\left(A_{R}, A_{R}\right)$. We will now give an example in which the two ideals $\Delta\left(A_{R}, A_{R}\right)$ and $\Sigma\left(A_{R}, A_{R}\right)$ are not the two ideals $I / a R$ and $K / a R$, different from what happens for biuniform modules.

Example 4.13. Let $R$ be a local commutative integral domain and $a$ a prime element of $R$ that does not generate the maximal ideal of $R$. For instance, $R$ could be the ring of polynomials $k[x, y]$ with $k$ a field and $x, y$ commutative indeterminates localized at the maximal ideal $(x, y)$ of $k[x, y]$, and $a$ could be the element $x$. We will prove that, in this case, one has $I / a R=K / a R=$ $\Sigma\left(A_{R}, A_{R}\right)$ and $\Delta\left(A_{R}, A_{R}\right)=0$.

As $R$ is commutative, we have that $E=R$ in the notation above so that $\operatorname{End}_{R}(R / a R)=R / a R$ is a local ring with maximal ideal $J(R) / a R=K / a R$. In particular, $K / a R \supseteq I / a R$ and $K / a R \supseteq \Sigma\left(A_{R}, A_{R}\right)$. But if $r \in K$, then $r a \in a J(R)$ so that $r \in I$. Thus $I / a R=K / a R$. Now if $r \in K=I=J(R)$, then multiplication by $r$ maps $R / a R$ into $J(R) / a R$. Thus the image of the endomorphism of $R / a R$ given by multiplication by $r$ is superfluous. This proves that $r+a R \in \Sigma(R / a R, R / a R)$ so that $K / a R=\Sigma\left(A_{R}, A_{R}\right)$. Finally, the endomorphism ring $R / a R$ of $R / a R$ is a commutative domain because $a$ is a prime element of the domain $R$. It follows that every non-zero endomorphism of $R / a R$ is injective. Thus $\Delta\left(A_{R}, A_{R}\right)=0$.

\section{From one ideal to $n$ ideals}

We will now show how it is possible to pass in Theorem 2.4 from the case of one ideal $\mathcal{I}$ to the case of $n \geq 2$ ideals $\mathcal{I}_{1}, \ldots, \mathcal{I}_{n}$. The case of two ideals was the object of study in [7]. We are grateful to Manuel Reyes, who suggested us Proposition 5.2 and Theorem 5.3 for the case when $n=2$.

We begin this section with an explicit presentation of some non-commutative polynomials with coefficients in the $\operatorname{ring} \mathbb{Z}$ of integers. Let $x, y_{1}, y_{2}, y_{3}, \ldots$ be infinitely many non-commutative indeterminates over the ring $\mathbb{Z}$ so that there is a strictly ascending chain

$$
\mathbb{Z}\left\langle x, y_{1}\right\rangle \subset \mathbb{Z}\left\langle x, y_{1}, y_{2}\right\rangle \subset \mathbb{Z}\left\langle x, y_{1}, y_{2}, y_{3}\right\rangle \subset \cdots
$$

of non-commutative integral domains. Here, $\mathbb{Z}\left\langle x, y_{1}, \ldots, y_{n}\right\rangle$ denotes the ring of polynomials in the non-commutative indeterminates $x, y_{1}, \ldots, y_{n}$ with coefficients in $\mathbb{Z}$.

Proposition 5.1. Let $x, y_{1}, y_{2}, \ldots$ be non-commutative indeterminates over the ring $\mathbb{Z}$ of integers and let $\mathbb{Z}\left\langle x, y_{1}, \ldots, y_{n}\right\rangle$ be the ring of non-commutative polynomials in the indeterminates $x, y_{1}, \ldots, y_{n}$ with coefficients in $\mathbb{Z}$ for every 
$n \geq 1$. Then for every $n \geq 1$, there is a unique polynomial $p_{n}=p_{n}\left(x, y_{1}, \ldots\right.$, $\left.y_{n}\right) \in \mathbb{Z}\left\langle x, y_{1}, \ldots, y_{n}\right\rangle$ such that

$$
1-p_{n} x=\left(1-y_{1} x\right)\left(1-y_{2} x\right) \cdots\left(1-y_{n} x\right) .
$$

Moreover, these polynomials $p_{n}, n \geq 1$, have the following properties:

(a) $1-x p_{n}=\left(1-x y_{1}\right)\left(1-x y_{2}\right) \cdots\left(1-x y_{n}\right)$ for every $n \geq 1$.

(b) $p_{1}=y_{1}$ and $p_{n+1}=y_{n+1}+p_{n}\left(1-x y_{n+1}\right)$ for every $n \geq 1$.

(c)

$$
\begin{aligned}
p_{n}= & \sum_{1 \leq i \leq n} y_{i}-\sum_{1 \leq i_{1}<i_{2} \leq n} y_{i_{1}} x y_{i_{2}} \\
& +\sum_{1 \leq i_{1}<i_{2}<i_{3} \leq n} y_{i_{1}} x y_{i_{2}} x y_{i_{3}}-\cdots+(-1)^{n-1} y_{1} x y_{2} x \cdots x y_{n}
\end{aligned}
$$

for every $n \geq 1$.

Proof. Such a polynomial $p_{n} \in \mathbb{Z}\left\langle x, y_{1}, \ldots, y_{n}\right\rangle$ exists because the product on the right in equation (1) is of the form " $1+$ monomials that terminate with $x "$. It is unique because $\mathbb{Z}\left\langle x, y_{1}, \ldots, y_{n}\right\rangle$ is an integral domain.

(a) If we multiply equation (1) by $x$ on the left, then we get that

$$
\begin{aligned}
x\left(1-p_{n} x\right) & =x\left(1-y_{1} x\right)\left(1-y_{2} x\right) \cdots\left(1-y_{n} x\right) \\
& =\left(x-x y_{1} x\right)\left(1-y_{2} x\right) \cdots\left(1-y_{n} x\right) \\
& =\left(1-x y_{1}\right) x\left(1-y_{2} x\right) \cdots\left(1-y_{n} x\right) \\
& =\left(1-x y_{1}\right)\left(1-x y_{2}\right) x \cdots\left(1-y_{n} x\right) \\
& =\cdots \\
& =\left(1-x y_{1}\right)\left(1-x y_{2}\right) \cdots\left(1-x y_{n}\right) x .
\end{aligned}
$$

But $x\left(1-p_{n} x\right)=x-x p_{n} x=\left(1-x p_{n}\right) x$ so that the identity in (a) holds because $x$ is a non-zero element of the integral domain $\mathbb{Z}\left\langle x, y_{1}, \ldots, y_{n}\right\rangle$.

(b) From the definition of $p_{1}$, we have that $1-p_{1} x=1-y_{1} x$, so that $p_{1}=y_{i}$. From the definition of $p_{n+1}$, we have that $1-p_{n+1} x=\left(1-y_{1} x\right) \cdots(1-$ $\left.y_{n+1} x\right)=\left(1-p_{n} x\right)\left(1-y_{n+1} x\right)=1-p_{n} x-y_{n+1} x+p_{n} x y_{n+1} x$ from which $p_{n+1}=p_{n}+y_{n+1}-p_{n} x y_{n+1}=y_{n+1}+p_{n}\left(1-x y_{n+1}\right)$.

(c) follows from equation (1).

The polynomials $p_{n}=p_{n}\left(x, y_{1}, \ldots, y_{n}\right)$ can also be viewed as elements of the path algebra of the quiver with two vertices $A$ and $B$, one arrow from $A$ to $B$ indexed by $x$ and $n$ arrows from $B$ to $A$ indexed by $y_{1}, y_{2}, \ldots, y_{n}$.

Proposition 5.2. Let $\mathcal{A}$ be a preadditive category, $\mathcal{I}_{1}, \ldots, \mathcal{I}_{n}$ be ideals of $\mathcal{A}$ and $f: A \rightarrow B$ be a morphism in $\mathcal{A}$. Assume that the image $\bar{f}: A \rightarrow B$ of $f$ in the factor category $\mathcal{A} / \mathcal{I}_{i}$ is an isomorphism for every $i=1,2, \ldots, n$, and let $g_{i}: B \rightarrow A$ be a morphism in $\mathcal{A}$ whose image in $\mathcal{A} / \mathcal{I}_{i}$ is the inverse of $\bar{f}$, $i=1,2, \ldots, n$. Then the image of $f$ in $\mathcal{A} / \mathcal{I}_{1} \cap \cdots \cap \mathcal{I}_{n}$ is an isomorphism and its 
inverse in $\mathcal{A} / \mathcal{I}_{1} \cap \cdots \cap \mathcal{I}_{n}$ is the image of the morphism $p_{n}\left(f, g_{1}, \ldots, g_{n}\right): B \rightarrow$ A.

Proof. We must prove that $1_{A}-p_{n}\left(f, g_{1}, \ldots, g_{n}\right) f \in \mathcal{I}_{i}(A, A)$ and $1_{B}-f p_{n}(f$, $\left.g_{1}, \ldots, g_{n}\right) \in \mathcal{I}_{i}(B, B)$ for all $i=1,2, \ldots, n$. Now $1_{A}-p_{n}\left(f, g_{1}, \ldots, g_{n}\right) f=$ $\left(1_{A}-g_{1} f\right)\left(1_{A}-g_{2} f\right) \cdots\left(1_{A}-g_{n} f\right)$ by equation $(1)$, and $1_{A}-g_{i} f \in \mathcal{I}_{i}(A, A)$ so that $1_{A}-p_{n}\left(f, g_{1}, \ldots, g_{n}\right) f \in \mathcal{I}_{i}(A, A)$ for all $i$. Similarly for $1_{B}-f p_{n}\left(f, g_{1}, \ldots\right.$, $\left.g_{n}\right)$ making use of the identity in Proposition 5.1(a).

Theorem 5.3. Let $\mathcal{I}_{1}, \ldots, \mathcal{I}_{n}$ be ideals of a preadditive category $\mathcal{A}$ with Jacobson radical $\mathcal{J}$. The following conditions are equivalent:

(a) The canonical functor $\mathcal{A} \rightarrow \mathcal{A} / \mathcal{I}_{1} \times \cdots \times \mathcal{A} / \mathcal{I}_{n}$ is local.

(b) The canonical functor $\mathcal{A} \rightarrow \mathcal{A} / \mathcal{I}_{1} \cap \cdots \cap \mathcal{I}_{n}$ is local.

(c) $\mathcal{I}_{1} \cap \cdots \cap \mathcal{I}_{n} \subseteq \mathcal{J}$.

Proof. (a) $\Rightarrow$ (b) The canonical functor $\mathcal{A} \rightarrow \mathcal{A} / \mathcal{I}_{1} \times \cdots \times \mathcal{A} / \mathcal{I}_{n}$ can be factored as the composite functor of the canonical functor $\mathcal{A} \rightarrow \mathcal{A} / \mathcal{I}_{1} \cap \cdots \cap \mathcal{I}_{n}$ and the canonical functor $\mathcal{A} / \mathcal{I}_{1} \cap \cdots \cap \mathcal{I}_{n} \rightarrow \mathcal{A} / \mathcal{I}_{1} \times \cdots \times \mathcal{A} / \mathcal{I}_{n}$.

(b) $\Rightarrow$ (c) The kernel of every local functor is contained in the Jacobson radical.

(c) $\Rightarrow$ (a) Assume $\mathcal{I}_{1} \cap \cdots \cap \mathcal{I}_{n} \subseteq \mathcal{J}$. Let $f: A \rightarrow B$ be a morphism in $\mathcal{A}$ whose image in $\mathcal{A} / \mathcal{I}_{1} \times \cdots \times \mathcal{A} / \mathcal{I}_{n}$ is an isomorphism so that all its images in the factor categories $\mathcal{A} / \mathcal{I}_{i}$ are isomorphisms. By Proposition 5.2, the image of $f$ in $\mathcal{A} / \mathcal{I}_{1} \cap \cdots \cap \mathcal{I}_{n}$ is an isomorphism. As $\mathcal{I}_{1} \cap \cdots \cap \mathcal{I}_{n} \subseteq \mathcal{J}$, the image of $f$ in $\mathcal{A} / \mathcal{J}$ is an isomorphism. But isomorphisms modulo the Jacobson radical are isomorphisms so that $f$ is an isomorphism of $\mathcal{A}$.

From Theorems 2.4 and 5.3, we obtain that:

Corollary 5.4. Let $\mathcal{I}_{1}, \ldots, \mathcal{I}_{n}$ be ideals of a preadditive category $\mathcal{B}$. Let $\mathcal{C}$ be the full subcategory of $\mathcal{B}$ whose objects are the objects $A$ of $\mathcal{B}$ with $\mathcal{I}_{1}(A, A) \cap$ $\cdots \cap \mathcal{I}_{n}(A, A) \subseteq J\left(\operatorname{End}_{\mathcal{B}}(A)\right)$. Then on the category $\mathcal{C}$, the ideal $\mathcal{I}_{1} \cap \cdots \cap \mathcal{I}_{n}$ is contained in the Jacobson radical $\mathcal{J}$ so that the canonical functor $\mathcal{C}: \mathcal{C} \rightarrow \mathcal{C} / \mathcal{I}_{1} \times$ $\cdots \times \mathcal{A} / \mathcal{I}_{n}$ is local. Moreover, the category $\mathcal{C}$ is the largest full subcategory of $\mathcal{B}$ with this property. Finally, if $\mathcal{B}$ is an additive category, then $\mathcal{C}$ is an additive category, and if $\mathcal{B}$ is additive and idempotents split in $\mathcal{B}$, then idempotents split also in $\mathcal{C}$.

Remark 5.5. From Theorem 5.3, it is also possible to obtain a very quick proof of [7, Proposition 3.1(b)] as follows. The statement of the proposition says that if $\mathcal{A}$ is a preadditive semilocal category, $\left(\mathcal{I}_{1}, \mathcal{I}_{2}\right)$ is a pair of ideals of $\mathcal{A}$ and every maximal ideal of $\mathcal{A}$ contains either $\mathcal{I}_{1}$ or $\mathcal{I}_{2}$, then the canonical functor $\mathcal{A} \rightarrow \mathcal{A} / \mathcal{I}_{1} \times \mathcal{A} / \mathcal{I}_{2}$ is local.

To prove this making use of Theorem 5.3, recall that the Jacobson radical of a semilocal category is the intersection of all maximal ideals [6, Theorem 4.8(1)]. If every maximal ideal of $\mathcal{A}$ contains either $\mathcal{I}_{1}$ or $\mathcal{I}_{2}$, then $\mathcal{I}_{1} \cap \mathcal{I}_{2}$ is contained 
in the Jacobson radical of $\mathcal{A}$, and therefore, the canonical functor $\mathcal{A} \rightarrow \mathcal{A} / \mathcal{I}_{1} \times$ $\mathcal{A} / \mathcal{I}_{2}$ is local by Theorem 5.3 .

Let $\mathcal{C}$ be a semilocal category and $\operatorname{Max}(\mathcal{C})$ be the collection of all its maximal ideals. For every object $A$ in $\mathcal{C}$, there exist finitely many maximal ideals $\mathcal{M}_{1}, \ldots, \mathcal{M}_{n}(n \geq 0)$ of $\mathcal{C}$ such that, for every maximal ideal $\mathcal{M}$ in $\mathcal{C}, A$ is a non-zero object in $\mathcal{C} / \mathcal{M}$ if and only if $\mathcal{M}=\mathcal{M}_{i}$ for some $i \in\{1, \ldots, n\}$. It follows that there is a functor $F: \mathcal{C} \rightarrow \oplus_{\mathcal{M} \in \operatorname{Max}(\mathcal{C})} \mathcal{C} / \mathcal{M}$ induced by the collection of canonical functors $\mathcal{C} \rightarrow \mathcal{C} / \mathcal{M}, \mathcal{M} \in \operatorname{Max}(\mathcal{C})$. This functor $F$ is isomorphism reflecting [6, Theorem 4.8]. From Proposition 5.2, we get that:

Proposition 5.6. Let $\mathcal{C}$ be a semilocal category. Then the canonical functor $F: \mathcal{C} \rightarrow \oplus_{\mathcal{M} \in \operatorname{Max}(\mathcal{C})} \mathcal{C} / \mathcal{M}$ is a local functor.

Proof. Let $f: A \rightarrow B$ be an isomorphism in $\mathcal{C}$ that becomes an isomorphism in $\mathcal{C} / \mathcal{M}$ for every maximal ideal $\mathcal{M}$ of $\mathcal{C}$. There exist finitely many maximal ideals $\mathcal{M}_{1}, \ldots, \mathcal{M}_{n}$ such that $A=B=0$ in $\mathcal{C} / \mathcal{M}$ for every maximal ideal $\mathcal{M} \in \operatorname{Max}(\mathcal{C}) \backslash\left\{\mathcal{M}_{1}, \ldots, \mathcal{M}_{n}\right\}$. For each $i=1,2, \ldots, n$, let $g_{i}: B \rightarrow A$ be a morphism in $\mathcal{C}$ that becomes the inverse of $f$ in $\mathcal{C} / \mathcal{M}_{i}$. By Proposition 5.2, the image of $f$ is an isomorphism in $\mathcal{A} / \mathcal{M}_{1} \cap \cdots \cap \mathcal{M}_{n}$ and its inverse in $\mathcal{A} / \mathcal{M}_{1} \cap \cdots \cap \mathcal{M}_{n}$ is the image of the morphism $p_{n}\left(f, g_{1}, \ldots, g_{n}\right): B \rightarrow A$. Thus $1_{A}-p_{n}\left(f, g_{1}, \ldots, g_{n}\right) f \in \mathcal{M}_{i}(A, A)$ for every $i=1,2, \ldots, n$. Also, $1_{A}-p_{n}\left(f, g_{1}, \ldots, g_{n}\right) f \in \mathcal{M}(A, A)$ for $\mathcal{M} \in \operatorname{Max}(\mathcal{C}) \backslash\left\{\mathcal{M}_{1}, \ldots, \mathcal{M}_{n}\right\}$ because $\mathcal{M}(A, A)=\operatorname{End}_{\mathcal{C}}(A)$. Thus $1_{A}-p_{n}\left(f, g_{1}, \ldots, g_{n}\right) f$ is in the intersection of all $\mathcal{M}(A, A)$ 's, which is the Jacobson radical of $\operatorname{End}_{\mathcal{C}}(A)$. Therefore, $p_{n}\left(f, g_{1}, \ldots, g_{n}\right) f$ is an automorphism of $A$ and $f$ is left invertible in $\mathcal{C}$. Similarly, from $1_{B}-f p_{n}\left(f, g_{1}, \ldots, g_{n}\right)$, we get that $f$ is right invertible. Hence $f$ is an automorphism of $A$ in $\mathcal{C}$.

\section{References}

[1] A. Alahmadi and S. K. Jain, A note on almost injective modules, Math. J. Okayama Univ. 51 (2009), 101-109.

[2] B. Amini, A. Amini, and A. Facchini, Equivalence of diagonal matrices over local rings, J. Algebra 320 (2008), no. 3, 1288-1310.

[3] A. Facchini, Module Theory, Endomorphism rings and direct sum decompositions in some classes of modules, Birkhäuser Verlag, Basel, 1998.

[4] - Representations of additive categories and direct-sum decompositions of objects, Indiana Univ. Math. J. 56 (2007), no. 2, 659-680.

[5] - A characterization of additive categories with the Krull-Schmidt property, Algebra and its applications, 125-129, Contemp. Math., 419, Amer. Math. Soc., Providence, RI, 2006.

[6] A. Facchini and M. Perone, Maximal ideals in preadditive categories and semilocal categories, J. Algebra Appl. 10 (2011), no. 1, 1-27.

[7] - On some noteworthy pairs of ideals in Mod- $R$, to appear in Appl. Categ. Structures, 2012.

[8] A. Facchini and P. Př́ihoda, Endomorphism rings with finitely many maximal right ideals, Comm. Algebra 39 (2011), no. 9, 3317-3338. 
[9] (2011), no. 3, 545-570

[10] K. R. Goodearl and R. B. Warfield, Jr., An Introduction to Noncommutative Noetherian Rings, Second Edition, London Math. Soc. Lecture Note Series 61, Cambridge Univ. Press, Cambridge, 2004.

[11] S. H. Mohamed and B. J. Müller, Continuous and Discrete Modules, London Math. Soc. Lecture Note Series 147, Cambdridge Univ. Press, Cambridge, 1990.

Adel Alahmadi

Department of Mathematics

FACULTY OF SCIENCE

King AbDulaziz University

JEDDAH 21589, SAUDi ARABIA

E-mail address: analahmadi@kau.edu.sa

Alberto FacChini

Dipartimento Di Matematica

Università di Padova, 35121 Padova, Italy

E-mail address: facchini@math.unipd.it 